\title{
Information flow principles for plasticity in foraging robot swarms
}

\author{
Lenka Pitonakova $^{1}$. Richard Crowder ${ }^{1}$. \\ Seth Bullock ${ }^{2}$
}

Received: 20 May 2015 / Accepted: 6 January 2016 / Published online: 9 February 2016

(C) The Author(s) 2016. This article is published with open access at Springerlink.com

\begin{abstract}
An important characteristic of a robot swarm that must operate in the real world is the ability to cope with changeable environments by exhibiting behavioural plasticity at the collective level. For example, a swarm of foraging robots should be able to repeatedly reorganise in order to exploit resource deposits that appear intermittently in different locations throughout their environment. In this paper, we report on simulation experiments with homogeneous foraging robot teams and show that analysing swarm behaviour in terms of information flow can help us to identify whether a particular behavioural strategy is likely to exhibit useful swarm plasticity in response to dynamic environments. While it is beneficial to maximise the rate at which robots share information when they make collective decisions in a static environment, plastic swarm behaviour in changeable environments requires regulated information transfer in order to achieve a balance between the exploitation of existing information and exploration leading to acquisition of new information. We give examples of how information flow analysis can help designers to decide on robot control strategies with relevance to a number of applications explored in the swarm robotics literature.
\end{abstract}

Keywords Swarm robotics · Foraging $\cdot$ Communication $\cdot$ Self-organisation $\cdot$ Plasticity

\section{Introduction}

Plasticity in a robot swarm is the ability of the robots to repeatedly organise and reorganise in response to changing demand characteristics. As such, it is central to the ability of a robot

Electronic supplementary material The online version of this article (doi:10.1007/s11721-016-0118-1) contains supplementary material, which is available to authorized users.

\footnotetext{
$凶$ Lenka Pitonakova

contact@lenkaspace.net

1 Institute for Complex Systems Simulation and Department of Electronics and Computer Science, University of Southampton, Southampton, UK

2 School of Computer Science, University of Bristol, Bristol, UK
} 
swarm to cope with real-world tasks when they are extended in time or space, since these typically feature inherent dynamism in the demand characteristics that the swarm must deal with. In this paper, we study the emergence of swarm plasticity in the context of swarm foraging with homogeneous simulated swarms that need to respond to changing resource deposits. We develop an information flow measure that allows us to understand the swarm's self-organisation and present robot behavioural strategies that lead to plasticity at the level of the entire swarm.

Swarm foraging is a behaviour that has received a considerable amount of attention in swarm robotics (e.g. Krieger and Billeter 2000; Liu et al. 2007; Gutiérrez et al. 2010). Robots must find and collect resources distributed throughout their environment. While designing strategies for swarm foragers is challenging, such strategies allow swarms of robots to coordinate their exploitation of the environment and to potentially forage more effectively. Furthermore, foraging is often used as a paradigm for studying other swarm behaviours, such as task allocation (e.g. Jevtic et al. 2012) or labour division (e.g. Zahadat et al. 2013), where worksites need to be searched for in the environment and work is distributed among the robots, or agent dispersion (e.g. Ranjbar-Sahraei et al. 2012), where robots need to coordinate their movement via communication. We choose foraging as a test bed application in this paper so that our results are relevant to a spectrum of topics covered in the current swarm robotics literature.

If we could create swarms that are not only able to perform given tasks, but able to perform them autonomously and reliably over a prolonged period of time in changeable environments, robot swarms could collect garbage from our streets, extract and gather minerals or be used in logistic applications. However, it is not their ability to forage itself, but rather their ability to adapt that makes swarm foraging systems interesting and useful (e.g. Dai 2009; Ducatelle et al. 2011). Therefore, if robot swarms are to address real-life applications, we need to understand how swarm intelligence works in dynamic environments. In order to do this, we need to establish ways of analysing artificial swarms so that we can generalise findings from particular experiments and aim towards a framework or a set of design principles that can guide future research and engineering. Recent work on self-organisation in artificial swarm systems has shown an increasing demand for such generalisations (e.g. Parunak and Brueckner 2004; Serugendo et al. 2006; Winfield 2009).

In order to gain a detailed understanding of swarm foraging and identify what robot-level behaviour leads to swarm-level plasticity, we simulate and analyse foraging robot swarms of a gradually increasing complexity in a number of different environments. Our robots utilise recruitment, i.e. they inform each other about foraging locations through direct communication. Section 2 gives an overview of relevant aspects of social insect foraging behaviour and discusses state of the art in swarm robotics. We introduce our simulation environment and the robot control algorithm in Sect. 3 and evaluate performance of our robot swarms in simple static environments in Sect. 4. We then test the ability of swarms with various communication strategies to discover and discriminate between deposits of different quality in Sects. 5 and 6 , and we demonstrate the importance of a balance between exploration and exploitation in dynamic environments, where deposit quality changes over time. By measuring information value, which captures the direct effect of information flow on resource collection, we show in Sects. 7-9 that if information spreads slowly through swarms, they are likely to remain effective as foraging conditions change, due to their ability to balance information utilisation and acquisition. On the other hand, if information spreads quickly through swarms, while performance may be improved under specific environmental conditions, such swarms may also be less capable of plastic self-organisation. The information value measure can thus help designers to decide on robot behavioural parameters that lead to desired work modes 
for their swarms. Furthermore, in Sect. 9, we offer a set of swarm design principles focused on communication and discuss their relevance to a wider range of problems including swarm foraging from multiple sites in parallel, swarm self-regulation, emergent task allocation and evolutionary robotics.

\section{Background}

\subsection{Swarm foraging in nature}

The behaviour of swarms of foraging robots is often inspired by ants or bees. Colonies of these social insects demonstrate an incredible ability to self-organise when their environment changes and have therefore been studied by both biologists and engineers.

Ants use pheromone trails to indicate paths through the environment and to inform their nest mates about foraging locations (Sumpter and Beekman 2003; Arab et al. 2012). Pheromone paths that lead to better food sources and are used by more ants become stronger over time and attract more and more workers, while paths to inferior sources, that are not being reinforced frequently enough, gradually evaporate.

Bees are another example of animals that use recruitment when foraging. However, unlike ants, bees use direct signalling and have a designated area in the hive, called the dance floor, where they perform recruitment waggle dances for nest mates that are interested in foraging (von Frisch 1967; Seeley et al. 1991; Biesmeijer and De Vries 2001). While the length and strength of a waggle dance is related to the quality of a particular flower patch, the position and orientation of a bee on the dance floor relative to the sun encodes the location of a patch relative to the hive, allowing recruits to travel to specific advertised food sources. An individual's decisions about whether to waggle dance, forage or abandon a patch are affected by olfactory and taste information from nectar samples obtained from other foragers through trophallaxis after one bee sends a begging signal to another (De Marco and Farina 2003; Farina et al. 2005). For example, when a forager discovers that other bees are processing nectar of a much better quality, it abandons its own source faster and has a higher preference for the better source for a number of days (De Marco and Farina 2001).

Compared to ants, bees are generally better at achieving plasticity. For example, ants find it difficult to establish a new shorter route to a food source if an established trail already exists (Ribeiro et al. 2009). Furthermore, ants rely on pheromone evaporation, which takes time and makes diversion of foraging effort from a depleted to a new patch relatively slow. On the other hand, bees abandon patches based on individual decisions, making their responses faster when a patch is depleted (Sumpter and Beekman 2003). Bee colonies also have the ability to switch between patches when their relative quality changes (Seeley et al. 1991). It has been argued that trophallaxis plays an important role in dynamic environments, as it allows information about flower patch quality to spread through the whole hive within hours, while waggle dancing only affects bees that follow dances and is thus a slower communication method (Farina et al. 2005). Additional evidence suggests that returning scouts use stop signals to directly inhibit waggle dances of recruiters that advertise alternative sites (Seeley et al. 2012). Bee colonies also achieve flexibility through opportunistic scouting when a recruited forager gets lost due to errors in signal propagation during waggle dance (Seeley 1994) and through inspection, i.e. occasional re-evaluation of abandoned flower patches (Granovskiy et al. 2012). 


\subsection{Robot swarm foraging in static environments}

There is a considerable volume of research that concentrates on swarm foraging during which robots are solitary and do not communicate at all. Such swarms are often used in static environments to retrieve targets and bring them back to a designated location (e.g. Arkin 1992; Balch 1999; Ulam and Balch 2004).

Ant-inspired robots in simulated experiments can drop cues directly into the environment in order to help others reach items of interest (Drogoul and Ferber 1993). The use of pheromone and its evaporation and dispersion can also be simulated (Fujisawa et al. 2014). In the real world, robots that move on a phosphorescent floor can use LEDs to create glowing paths (Mayet et al. 2010). Alternatively, robots can deposit alcohol trails and use chemical sensors to follow them (Russell 1999; Fujisawa et al. 2014) or a centralised server can store virtual pheromone deposited by robots and use a projector to display it, allowing robots to follow pheromone trails using visual sensors (Kazama et al. 2005; Garnier et al. 2007). In order to avoid the difficulty of using pheromone-like substances that require a specific arena set-up, a virtual pheromone is often represented by designated stationary robots that communicate pheromone levels to others nearby (e.g. Hoff et al. 2013; Ducatelle et al. 2011). Similarly, in a set-up inspired by bee trophallaxis, a portion of the swarm is designated for propagation of values passed from robot to robot, allowing a gradient to be established between the base and a resource patch (Schmickl and Crailsheim 2008; Nouyan et al. 2009). It is also possible to use the whole swarm as a medium that holds pheromone paths, allowing virtual ants to travel through the robots and establish the shortest path to a resource (Campo et al. 2010).

Some ant-inspired (e.g. Krieger and Billeter 2000), as well as bee-inspired (e.g. Alers et al. 2011; Lee and Ahn 2011) robotic systems use direct signals that allow robots to recruit others to specific deposit locations when they arrive at the base. Alternatively, robots may communicate at any point when they meet each other during foraging (e.g. Valdastri et al. 2006; Gutiérrez et al. 2010; Miletitch et al. 2013). To localise themselves and the objects of interest, robots use path integration and usually store one vector pointing towards the base and one pointing towards a found deposit.

Bee-inspired algorithms have also been applied to help robots aggregate in areas of interest (Schmickl and Hamann 2010) or to optimise a swarm's energy intake from resource that was collected in the environment and processed in the base (Thenius et al. 2008). It is often difficult to perform experiments with large swarm sizes, to repeat experiments a sufficient number of times, or to collect statistics about robot behaviour during real-world experiments, causing many researches to rely on simulated data when a thorough analysis of swarm behaviour is required (e.g. Lee and Ahn 2011; Liu et al. 2007; Campo and Dorigo 2007; Miletitch et al. 2013).

\subsection{Robot swarm foraging in dynamic environments}

When foraging in environments that change over time, swarms need to possess some form of self-organisation on the level of the collective or adaptation at the individual level in order to cope with changing foraging conditions. Bees use a combination of both techniques. They adapt their response thresholds to various stimuli (Seeley 1994) and learn odours of profitable flowers (Farina et al. 2005). Colonies are also capable of self-organisation that results from evolved responses of bees to individual and social information (e.g. Seeley et al. 1991; De Marco and Farina 2003).

A frequently studied swarm robot behaviour is the ability of swarms to adjust the number of foragers and resting robots based on changing resource abundance. Robots can be equipped 
with means to perceive their own foraging performance and the performance of others and adapt their own control parameters accordingly (e.g. Campo and Dorigo 2007; Liu et al. 2007). Alternatively, self-organisation on the swarm level, that emerges when robots change their actions based on individual and social information, can also be used to make swarms adapt (e.g. Sarker and Dahl 2011).

In other experiments, foraging robots were required to distribute their foraging effort between different types of resource proportional to type abundance. It was shown that selforganising swarms could successfully solve this task if a correct balance between information sharing among robots and information acquisition by robots is achieved (e.g. Jones and Matarić 2003; Schmickl et al. 2007).

Finally, some foraging experiments involve bucket brigading, where robots form chains of adjacent "working areas" that run from the deposit location to the robot base, and robots move resources along these chains (e.g. Shell and Mataric 2006; Nouyan et al. 2009; Pini et al. 2013). An individual robot's working area can be adapted online to give a swarm the ability to follow mobile deposits (Lein and Vaughan 2009).

Here, in an approach similar to that of Jones and Matarić (2003), Schmickl et al. (2007), Sarker and Dahl (2011), robots do not adapt their individual control parameters, but share information with one another in order to achieve self-organisation at the level of the swarm.

\section{Methods}

\subsection{Simulation environment}

All the experiments reported here are performed in the ARGoS simulation environment (Pinciroli et al. 2012) using MarXbot robots (Bonani et al. 2010). ARGoS is a C++ environment with a realistic 3D physics engine that was specifically designed for program compatibility with MarXbots (also called foot-bots) and e-pucks. By using this type of simulation environment, we can model not only decision-making of robots, but also their physical interactions with each other and with the world. The physical aspect is important as it can lead to various interferences between robots and affect their performance, for example when multiple robots try to access a deposit at the same time or when they attempt to communicate with each other (Pitonakova et al. 2014).

The simulation takes place in continuous space and updates itself 10 times per second. The foraging arena is $50 \mathrm{~m} \times 50 \mathrm{~m}$ large and contains a centrally located circular base surrounded by resource deposits (Figs. 1, 2). A similar set-up has been used previously in simulated (e.g. Balch and Arkin 1994; Campo and Dorigo 2007) and real-world (e.g. Labella et al. 2004; Gutiérrez et al. 2010) experiments.

The base has a radius of three metres, and is divided into two sections: an interior circular dance floor and an annular unloading area around it (Fig. 1). A light source is placed above the middle of the base that the robots can use as a reference for navigation towards and away from the centre of the base (as in, for example, Krieger and Billeter 2000; Ferrante and Duéñez Guzmán 2013; Pini et al. 2013).

Resource is distributed throughout the environment in the form of a number, $N$, of discrete deposits. Each deposit is cylindrical, with radius $r_{D}$. The value of deposit $i$ to a robot is related to two properties: the volume of the deposit, $V_{i}$, which represents the gross amount of material it contains, and the quality of the deposit, $Q_{i}$, which represents how rich this material is. The total amount of resource in a deposit is thus $V_{i} \times Q_{i}$. Each robot loads a maximum volume 


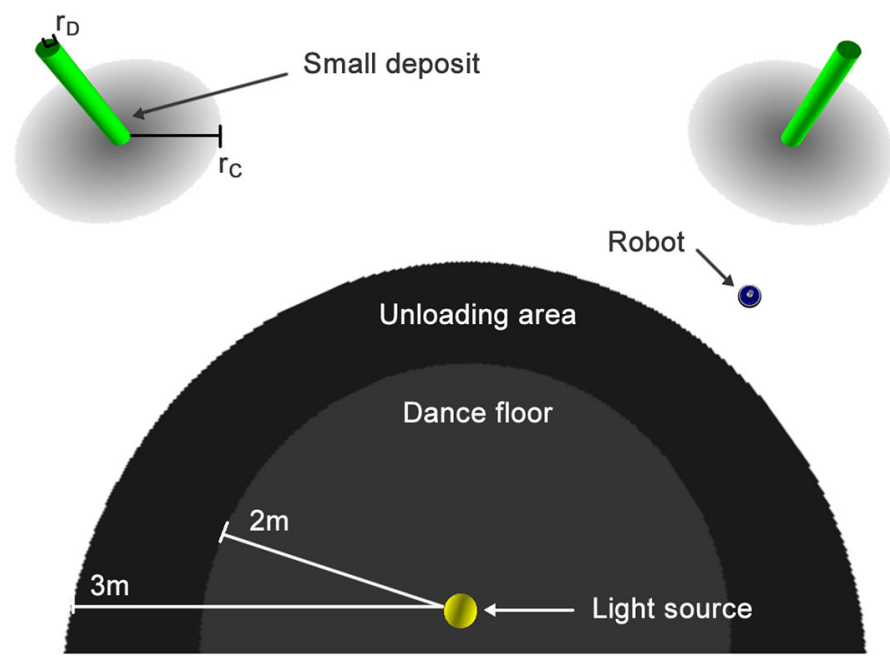

Fig. 1 ARGoS simulation screenshot of a base and scattered small deposits. The base consists of a circular dance floor with a radius of $2 \mathrm{~m}$, where recruitment takes place, and an unloading area that forms a ring around the dance floor where returning foragers drop collected material. There is also a light source above the centre of the base. The whole base has a radius of $3 \mathrm{~m}$. A robot with radius of $8.5 \mathrm{~cm}$ is located near the base. Each deposit has a colour gradient around it to allow nearby robots to navigate towards it (Color figure online)

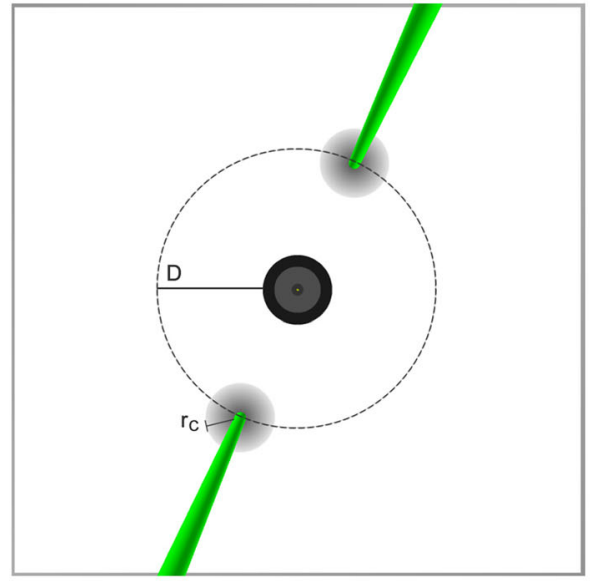

(a)

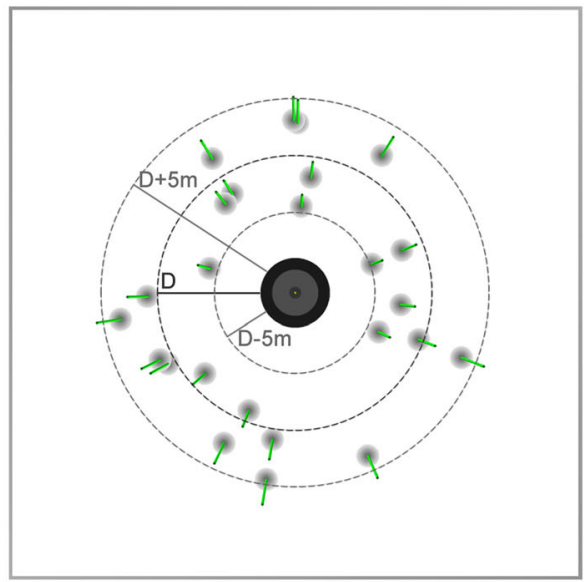

(b)

Fig. 2 ARGoS simulation screenshot of the experimental arena containing a base in the centre and deposits in the a Heap2 and b Scatter 25 scenarios with $D=9 \mathrm{~m}$. The deposits are represented as cylinders, with their heights corresponding to their respective volumes, and have colour gradients with radius $r_{C}$ around them to guide navigation of nearby robots (Color figure online)

of $L_{\max }$ units per foraging trip. The total amount of resource that a robot may load from deposit, $i$, in a single trip is thus $L=L_{\max } \times Q_{i}$ or $L=V_{i} \times Q_{i}$ if $V_{i}<L_{\max }$.

Consider two deposits, $A$ and $B$, with volumes greater than $L_{\max }$ and where deposit $A$ has twice the volume of $B$, but deposit $B$ has twice the quality of deposit $A$. Robots carrying all of deposit $A$ back to the base will have foraged the same amount of resource as robots 
carrying all of deposit $B$ back to the base because $V_{A} \times Q_{A}=V_{B} \times Q_{B}$, but robots foraging from deposit $A$ will have needed more trips to achieve this since $V_{A}>V_{B}$.

In the following environments, the volume of each deposit is $100 / N$ units, i.e. total volume of deposits is 100 units and is conserved across environments. $N$ is always selected so that $V_{i}$ is an integer. The default maximum value of $L$ is $L_{\max }=1$ unit, although $L_{\max }$ is reduced to 0.25 units in later simulations in order to increase the amount of time taken by swarms to complete the foraging task. The default value of $Q$ for all deposits is 1 , although some scenarios are specifically designed to contain deposits of varying quality.

In order to enable robots close to a deposit to move towards it, a colour gradient with radius $r_{C}$ is centred on the floor around each deposit. In a real-world experiment, navigation based on the colour gradient could be replaced by visual-based navigation, for instance.

There are two types of scenario (Fig. 2):

- $\operatorname{Heap} N: N$ deposits distributed evenly around the base at a distance $D=\{7,9,11,13\} \mathrm{m}$ from the base edge. These deposits represent heaps of resource that have large volumes and occupy a large area, with $r_{D}=0.5 \mathrm{~m}$ and $r_{C}=3 \mathrm{~m}$. For example, a Heap2 scenario contains two deposits with volume of 50 units each.

- Scatter $N$ : $N$ deposits randomly distributed between $(D-5) \mathrm{m}$ and $(D+5) \mathrm{m}$ from the base edge. These deposits are small, with $r_{D}=0.1 \mathrm{~m}$ and $r_{C}=1 \mathrm{~m}$, and often numerous, containing a small $V$ each. For example, a Scatter 25 scenario contains 25 deposits with volume of four units each.

A laden robot returning to the base deposits its load in the unloading area in the form of $N_{p}$ number of pellets of size $0.1 \mathrm{~m}^{3}$, where $N_{p}=L \times\left(4 \backslash L_{\max }\right)$, set to the closest larger integer. For example, when $L_{\max }=1$ and $L=1$, a robot deposits four pellets. When $L_{\max }=1$ and $L=0.1$, the robot deposits one pellet, etc. These pellets have the potential to cause congestion in the unloading area if many are deposited at the same time. Deposited pellets disappear from the simulation after a period of unloading area handling time, $t_{H}$, representing their use or consumption by a hypothetical unmodelled system of robots or human users. By setting $t_{H}=0 \mathrm{~s}$, the system is able to represent pellets that cause no congestion in the unloading area. Scenarios where $t_{H}>0 \mathrm{~s}$ are explored in Sect. 8.2.

\subsection{Robots}

The simulated marXbots (Bonani et al. 2010) are circular, differentially steered, robots with a diameter of $0.17 \mathrm{~m}$ that in our simulation can reach a maximum speed of $5 \mathrm{~cm} / \mathrm{s}$. They are equipped with four colour sensors pointed to the ground, a ring of 24 infrared proximity sensors used for collision avoidance, a light sensor used for navigation towards the base, a range and bearing module used for localisation of other robots and for communication, wheel-mounted sensors utilised for odometry and a ring of eight colour LEDs used for debugging. The maximum range at which the robots can detect objects via the proximity sensors is $0.3 \mathrm{~m}$. Their maximum communication range via the range and bearing module is $5 \mathrm{~m}$. The range and bearing module is based on line of sight, and hence intermittent ranging and communication problems are possible, although they do not have a significant effect in the scenarios we explore here. It is assumed in the simulation that the light sensor can detect the light above the base from anywhere in the experimental arena (as in, for example, Labella et al. 2004; Gutiérrez et al. 2010). Sensor noise and wheel slippage are not modelled. Consequently, there is no odometry error in our simulation.

The robot control algorithm is inspired by foraging bees, and the robots are modelled as finite state machines (Fig. 3). A robot starts in a random orientation and position on the 

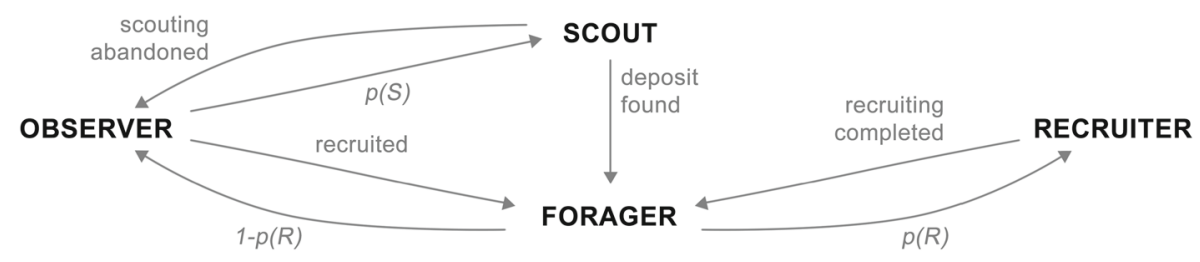

Fig. 3 Finite state machine representation of the robot controller

dance floor as an observer, ready to receive recruitment signals. Observers move randomly on the dance floor and avoid travelling into the unloading area. When a recruitment signal is received, the robot becomes a forager and navigates towards a deposit location obtained from the recruiter. Alternatively, an observer still on the dance floor can become a scout with scouting probability $p(S)=10^{-3}$ at each time step.

Scouts leave the base and use Lévy movement (Reynolds and Rhodes 2009) to search for a deposit within $20 \mathrm{~m}$ from the base. Any scout that cannot find a deposit within $600 \mathrm{~s}$ returns to the dance floor and becomes an observer. While outside the base, a robot updates its estimation of the relative position of the base using path integration based on odometry at each time step (e.g. Borenstein 1998; Lemmens et al. 2008; Gutiérrez et al. 2010). When a scout discovers a deposit, it becomes a forager. All foragers load $L$ units of volume of the resource and determine an estimate of the deposit's energy efficiency (after Seeley 1994):

$$
E_{E}=\frac{V_{i}^{\prime} \times Q_{i}}{d_{i}}
$$

where $V_{i}^{\prime}$ is the volume left in the deposit after the robot's visit, $Q_{i}$ is the deposit quality, and $d_{i}$ is the odometry-estimated linear distance from the unloading area to the deposit. The robot then returns back to the base utilising phototaxis and keeps track of its relative position to the deposit using odometry.

After a forager unloads its cargo in the unloading area, it moves to the dance floor and becomes a recruiter with a recruitment probability $p(R)$ :

$$
p(R)= \begin{cases}1.0 & \text { if } E_{E}>0 \\ 0.0 & \text { else }\end{cases}
$$

and performs recruitment inspired by bee waggle dancing for $T_{R}=120 \mathrm{~s}$, randomly moving across the dance floor and advertising its deposit location to all observers located within communication range $d_{C}$. In order to minimise the influence of the particular direction from which it arrived, and thereby give it the chance to influence more observers, the recruiter travels to the middle of the dance floor before it starts recruiting. Similarly, forager bees enter the dance floor from a single direction through a small nest entrance (Seeley and Morse 1976), allowing potential recruits to interact with any recruiter regardless of its previous foraging location.

The deposit location is communicated to each observer in a one-to-one fashion, where local axes of the robots and their alignment relative to each other are taken into account when conveying the positional information (Gutiérrez et al. 2010). While this technique is sensitive to the accuracy of a robot's sensors (Gutiérrez et al. 2010; Miletitch et al. 2013), it removes the need for a shared reference point. Note that bees, on the other hand, orient their dances relatively to the sun (von Frisch 1967). 
The recruiter always resumes foraging from the same deposit after it completes recruitment. In cases when there is no deposit to return to $(p(R)=0)$, a robot does not recruit or forage again but becomes an observer instead.

If a forager reaches a deposit location but the deposit cannot be found, the robot performs neighbourhood search that lasts 180 simulated seconds and during which it moves randomly in a circular area with a radius of $2 \mathrm{~m}$ around the expected deposit location. If the search is unsuccessful, the robot returns to the base to become an observer. Any unsuccessful foragers and scouts are opportunistic and start foraging from a deposit if they find one on their way back to the base.

\section{Foraging performance in static environments}

Before exploring scenarios that require plasticity, experiments were performed in Heap1, Heap2, Heap4, Scatter 10 and Scatter 25 scenarios that lasted $T=1$ simulated hour and where all deposits had a constant quality $Q=1$ throughout the simulation. We aimed to understand how the communication range, $d_{C}$, and the scouting probability, $p(S)$, of robots affected the total amount of resource collected in these scenarios. Each experiment was repeated 50 times. By varying $d_{C}$ and $p(S)$ to define three types of robot, we could encourage three types of homogeneous robot swarm: (Fig. 4):

- Solitary robots, where observers left the dance floor almost instantaneously to scout $\left(p(S)=10^{-1}\right)$, and robots could never recruit each other $\left(d_{C}=0 \mathrm{~m}\right)$

- Short-range recruiters, where observers spent longer on the dance floor $\left(p(S)=10^{-3}\right)$, but robots could only recruit observers that were near to them on the dance floor $\left(d_{C}=0.6 \mathrm{~m}\right)$

- Long-range recruiters, where robots could recruit any observer on the dance floor $\left(d_{C}=5 \mathrm{~m}, p(S)=10^{-3}\right)$

In the most extreme Heap scenario, Heap1, with only a single large deposit, using large $d_{C}$ and small $p(S)$ was the most beneficial as it allowed for mass recruitment once the deposit was discovered and foragers returned to the base to convey the deposit location to any unsuccessful scouts, making long-range recruiters the most suitable option (Fig. 4a). In the other extreme scenario, Scatter 25 , where there were 25 small deposits, solitary foraging led to the best results (Fig. 4b). In this case, recruitment damaged performance because of environmental interference between robots, where the robots altered each other's foraging

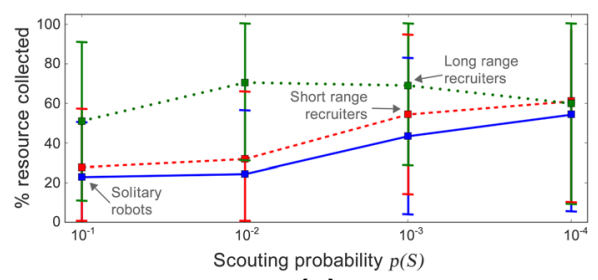

(a)

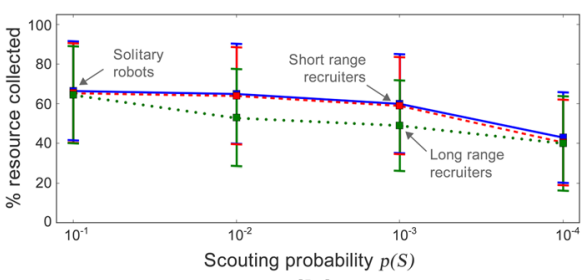

(b)

Fig. 4 Foraging performance of 25-robot swarms in the a Heap1 and $\mathbf{b}$ Scatter25 scenarios using various $p(S)$ values and $d_{C}=0 \mathrm{~m}$ (solid line), $d_{C}=0.6 \mathrm{~m}$ (dashed line) and $d_{C}=5 \mathrm{~m}$ (dotted line). Each point represents mean percentage of available resource that was collected in a given scenario, collated over 50 one-hour-long runs for each of the deposit distances in the set $D=\{7,9,11,13\} \mathrm{m}$. The whiskers represent $95 \%$ confidence intervals 
environment by depleting low-volume deposits and where recruitment thus led to a lot of wasteful foraging trips (see Pitonakova et al. 2014 for discussion of robot-robot interference during foraging). Short-range recruiters could achieve a balance between exploration and exploitation, which made them more robust across scenarios, although they never achieved performance better than that of the other two swarm types (see the dashed line in Fig. 4).

The results shown in Fig. 4 were obtained using swarms of 25 robots. Similar relationships between $d_{C}$ and $p(S)$ were found for swarms of 15 and 35 robots. The findings also held for less extreme environments such as Heap2, Heap4 or Scatter10, although results varied between runs more in these intermediate scenarios.

\section{Heterogeneous deposit quality}

Having established that recruitment is most beneficial in Heap scenarios, where deposits are large but hard to find, we now explore what type of information transfer would allow the robots to collectively choose a deposit of a better energy efficiency. We examined the following scenarios using deposit distances $D=\{7,9,11,13\} \mathrm{m}$ :

- Heap2A: two deposits with volume $V=50$ each and with qualities $Q_{1}=0.5, Q_{2}=1.5$

- Heap2B: two deposits with $V=50$ each and with qualities $Q_{1}=0.1, Q_{2}=1.9$

- Heap4A: four deposits with $V=25$ each and with qualities $Q_{1-3}=0.5, Q_{4}=2.5$

- Heap4B: four deposits with $V=25$ each and with qualities $Q_{1}=0.1, Q_{2}=0.5$, $Q_{3}=1.5, Q_{4}=1.9$

The maximum volume $L_{\max }$ loaded by a robot per foraging trip was decreased from 1.0 to 0.25 in all experiments reported below. The robots still collected $L_{\max } \times Q$ units of net resource value per foraging trip and deposited a maximum of four pellets in the unloading area. Decreasing $L_{\max }$ causes swarms to carry out more foraging trips during a simulation (see online supplementary material, Fig. S1), generating a larger data set which is necessary in order to analyse foraging dynamics when the environment changes over time.

The swarms used in the previous experiments were unable to discriminate between deposits based on energy efficiency as robots always followed the first recruiter that they met and remained foraging from a deposit until it was fully depleted. On average, such swarms thus distributed themselves to forage from all deposits equally. They were used as control swarms in the following experiments.

In order to allow robots to preferentially concentrate their foraging effort on deposits with higher energy efficiency, we created swarms of beggers, where all robot states and state transitions remained the same, but where recruiters asked each other about the energy efficiency of advertised deposits. If another robot's deposit had higher $E_{E}$, a recruiter switched to advertise this deposit and then foraged from it. This strategy was inspired by the begging behaviour of bees described in Sect. 2.1 $1^{1}$. As before, we tested beggers with both short-range $(0.6 \mathrm{~m})$ and long-range $(5 \mathrm{~m})$ communication signals.

Swarms of short-range beggers were able to identify and concentrate on better deposits within the first $20 \mathrm{~min}$ and could thus collect more resource than the control swarms (Fig. 5a). Long-range beggers switched to better deposits faster, making them more efficient at the task, especially for larger deposit distances (Fig. 5b). The size of the difference between deposit qualities did not affect the time it took to switch to a better deposit given a particular number

\footnotetext{
1 The begging behaviour of our robots is simplified compared to that of bees. Bees integrate information received from multiple nest mates, and their foraging preferences can be affected for a number of days (De Marco and Farina 2001; Farina et al. 2005).
} 


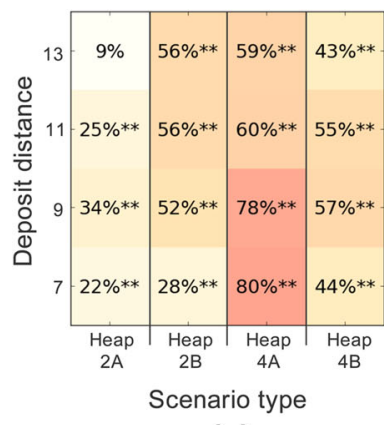

(a)

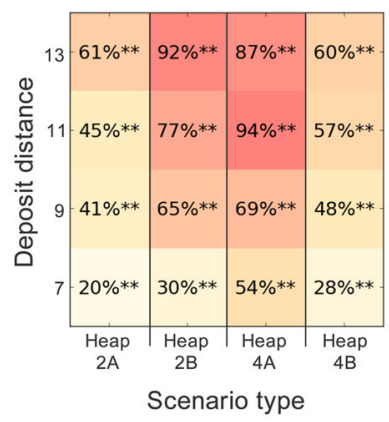

(b)

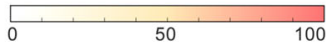

Fig. 5 Difference in the amount of resource collected compared to control (non-switching) swarms using 25 beggers with a short-range, b long-range signals during one simulated hour. The measured values represent averages from 50 independent runs. Statistically significant differences are indicated with asterisks (Wilcoxon signed-rank test, $* *=p<0.01, *=p<0.05$ )

of deposits. However, performance of both swarms was better in the Heap2B scenarios compared to Heap2A, as the difference between deposit qualities was larger in Heap2B and it was thus more advantageous to forage from the better deposit. Similarly, when robots could concentrate on a deposit with quality 2.5 in Heap4A, they performed better than in Heap4B, where the best deposit had quality of only 1.9 .

\section{The ability of robots to repeatedly switch between deposits}

In biological settings such as nectar foraging, the quality of foraging sites changes over time. In robotic applications, a preference for where to forage may also need to change due to the nature of the task, orders from humans, etc. It is thus important not only to be able to collectively select a better foraging site, but to be able to do so repeatedly, as the environment changes. Therefore, in dynamic environments, the ability to achieve a balance between exploitation and exploration becomes important.

To test the ability of our swarms to preferentially forage from better deposits in dynamic environments over a long period of time, we ran simulations over a number of hours and changed deposit qualities (but not their locations) at the end of each deposit quality change interval of length $T_{Q}$. When the change was made, deposits were assigned new qualities from a quality set given for a particular scenario and their volumes were replenished, so that the amount of available resource was the same at the beginning of each quality change interval.

In this task, strong commitment to a single best deposit could be a serious problem, as it might cause the swarm to loose track of other deposits and of new places to forage from when the quality change occurs. This was the case in all Heap2 scenarios, where beggers locked into foraging from a single deposit (Fig. 6a), an effect that was more pronounced for long-range beggers, and that held for both short $\left(T_{Q}=1 \mathrm{~h}\right)$ and long $\left(T_{Q}=2 \mathrm{~h}\right)$ quality change intervals.

On the other hand, in all Heap4 scenarios, where deposits had only half of the volume compared to the Heap2 scenarios and the differences between their energy efficiencies were thus smaller, the resulting foraging pattern depended on the communication range of robots and the length of the quality change interval used $\left(T_{Q}\right)$. Since beggers usually rapidly recruited each other to the best deposit found, large groups of robots quickly exploited a single foraging 


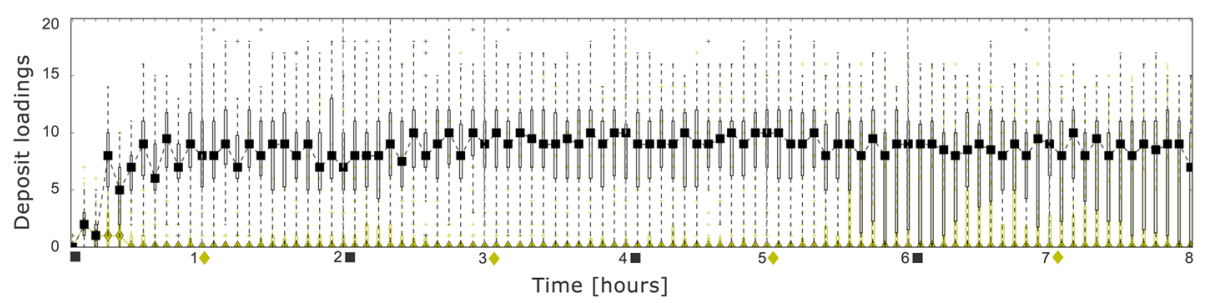

(a)

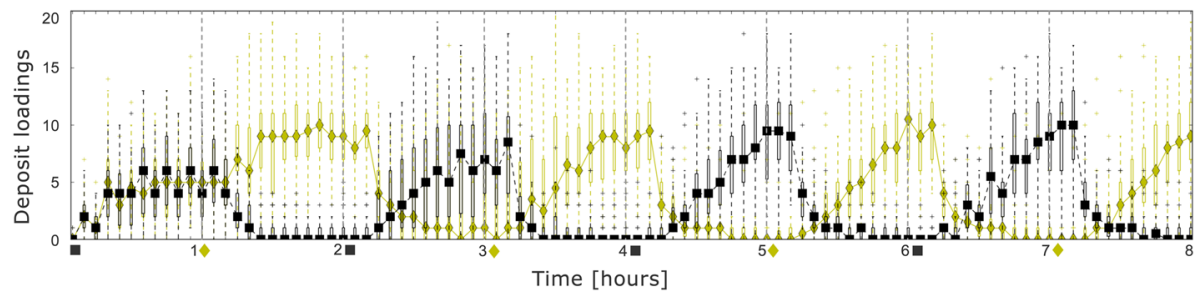

(b)

Fig. 6 Median number of loadings from two deposits (square and diamond symbols) during the first $8 \mathrm{~h}$ of simulation using $\mathbf{a} 25$ short-range beggers and $\mathbf{b} 25$ short-range checkers in the Heap2B scenario with $D=9 \mathrm{~m}$, $T_{Q}=1 \mathrm{~h}$. Deposit qualities were exchanged every hour during the experiment. The symbol of the deposit with higher quality is shown along the time axis at the beginning of each hour. Each data point represents a median value for a particular time interval and is based on a set of results collected from 50 independent runs. A data point is surrounded by a box, representing the inter-quartile range or "middle fifty" of the result set, and whiskers representing the "middle 97", with outliers outside this range shown as plus signs

site until its energy efficiency fell below that of alternative deposits, at which point the robots rapidly switched to a different deposit. This repeated every time a certain volume of a deposit that was currently being exploited by a majority of robots was depleted, preventing the swarms from preferentially foraging from deposits with superior quality and, on average, to forage from all deposits with an equal probability (Fig. 7a). Unlike in Heap2, some members of the swarm always foraged from inferior deposits and the swarm thus retained the memory of all deposits in the environment. However, the inability to forage from deposits of superior quality for sufficiently long caused short-range beggers when $T_{Q}=1 \mathrm{~h}$ and long-range beggers when $T_{Q}=1 \mathrm{~h}$ and $2 \mathrm{~h}$ to collect less resource that the control swarms (Figs. 8a, b, 9a, b). On the other hand, when $T_{Q}=2 \mathrm{~h}$, deposit quality remained stable long enough, allowing shortrange beggers to eventually choose the best deposit after a period of indecisiveness and more importantly to repeat this pattern during each quality change interval. This led to foraging performance better than that of the control swarms in both Heap4A and Heap4B scenarios when deposits were near the base (Fig. 9a).

These results suggested that a swarm behaviour where the spread of social information is regulated could potentially lead to more effective plastic reconfiguration and consequently more sustainable preferential foraging. To test this hypothesis, we introduced a modified behaviour that explicitly regulated the spread of social information. A swarm of checkers was implemented, where the robots did not compare deposit energy efficiency directly with other recruiters, as it was the case with beggers. However, instead of having a binary recruitment probability, $p(R)$ (Eq. 2), and a fixed recruitment time, $T_{R}$, checkers calculated these variables based on how the energy efficiency of a deposit had changed since the last time they had visited it: 


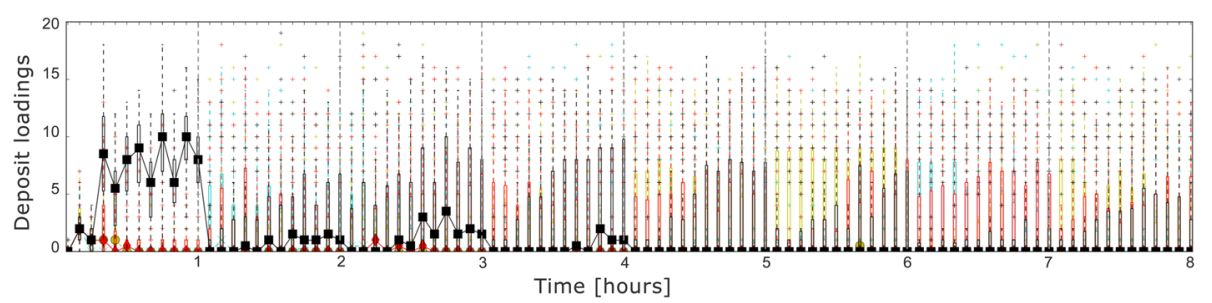

(a)

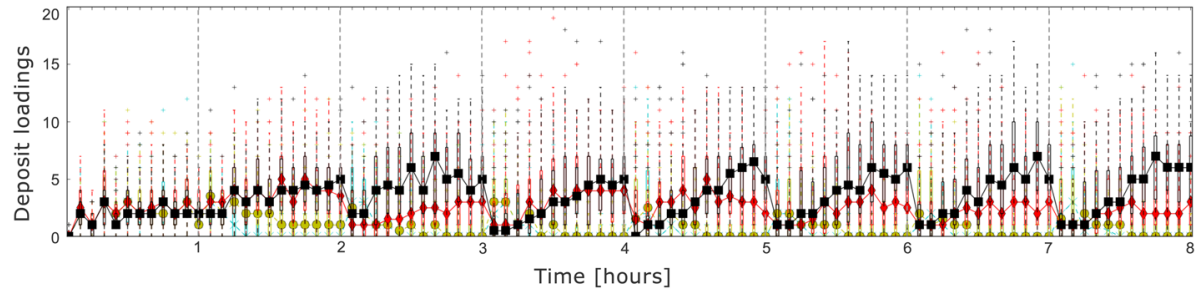

(b)

Fig. 7 Median number of loadings from four deposits during the first $8 \mathrm{~h}$ of simulation using a 25 short-range beggers and b 25 short-range checkers in the Heap4B scenario with $D=9 \mathrm{~m}, T_{Q}=1 \mathrm{~h}$, based on 50 independent runs. Deposit qualities were assigned randomly every hour during the experiment. Therefore, instead of identifying loadings from a particular deposit in a particular location, like in Fig. 6, each symbol identifies loadings from a deposit of a particular quality in a given time interval: $Q=1.9$ (squares), $Q=1.5$ (diamonds), $Q=0.5$ (circles), $Q=0.1$ (crosses)

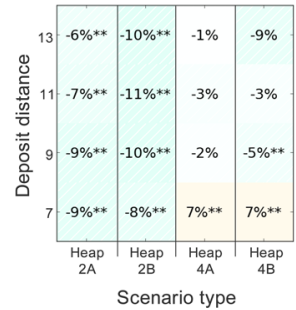

(a)

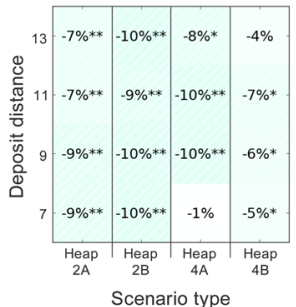

(b)

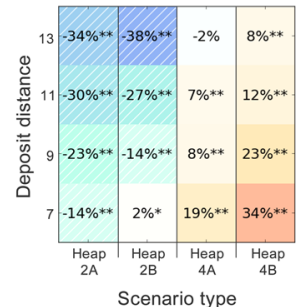

(c)

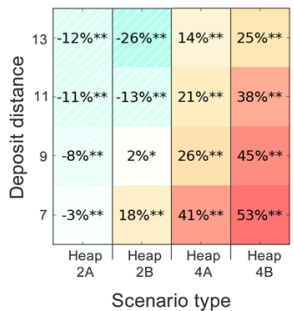

(d)

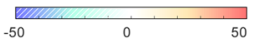

Fig. 8 Difference in the amount of resource collected compared to control (non-switching) swarms using a 25 short-range beggers, $\mathbf{b} 25$ long-range beggers, $\mathbf{c} 25$ short-range checkers, $\mathbf{d} 25$ long-range checkers during $1 \mathrm{~h}<T<13 \mathrm{~h}$ of 50 independent runs, using $T_{Q}=1 \mathrm{~h}$. Statistically significant differences are indicated with asterisks (Wilcoxon signed-rank test, $* *=p<0.01, *=p<0.05$ )

$$
\delta=\min \left(1.0, \frac{E_{E}}{E_{E}^{\prime}} \delta^{\prime}\right)
$$

where $E_{E}^{\prime}$ and $\delta^{\prime}$ were the energy efficiency of the deposit and $\delta$ measured during the last loading event,

$$
\begin{aligned}
p(R) & =\delta \\
T_{R} & =\delta \times 120 s
\end{aligned}
$$

Because their recruitment probability, and consequently their probability of returning to a deposit, decreased gradually as the deposit became depleted, checkers abandoned a foraging site one by one over time, allowing them to be recruited by other robots or to go scouting. 


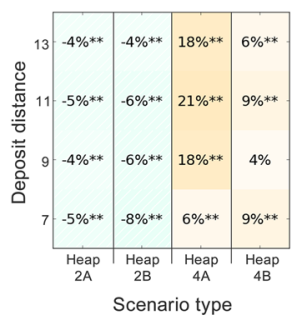

(a)

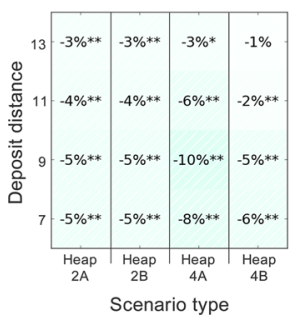

(b)

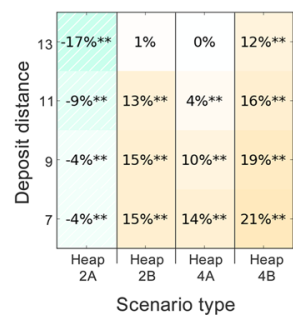

(c)

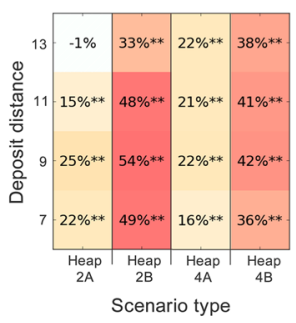

(d)

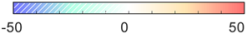

Fig. 9 Difference in the amount of resource collected compared to control (non-switching) swarms using a 25 short-range beggers, b 25 long-range beggers, c 25 short-range checkers, $\mathbf{d} 25$ long-range checkers during $2 \mathrm{~h}<T<26 \mathrm{~h}$ of 50 independent runs, using $T_{Q}=2 \mathrm{~h}$. Statistically significant improvements are indicated with asterisks (Wilcoxon signed-rank test, $* *=p<0.01, *=p<0.05$ )

By contrast, both the control swarms and beggers remained foraging from the same deposit until it became completely depleted or until, in the case of beggers, the robots received social information about a better deposit. Perhaps more importantly, the fact that checkers evaluated the change in energy efficiency of a deposit each time they visited it, enabled them to immediately abandon a deposit if its quality suddenly decreased, without having to rely on receiving knowledge from other robots about better deposits, as the beggers did.

The checker behavioural strategy was also inspired by bees, in particular by their food patch abandonment behaviour (Seeley 1994; Biesmeijer and De Vries 2001). An agentbased model, where recruitment time was influenced by quality of an advertised site but where agents, similarly to our beggers, could also switch to another site after recruitment was explored by Valentini et al. (2014).

The checker strategy allowed the swarms to repeatedly switch to a better deposit in both Heap2 (Fig. 6b) and Heap4 (Fig. 7b) scenarios, unlike the beggers that either foraged from one deposit throughout the whole run or were often unable to make a clear collective decision. The checker swarms were able to concentrate on exploiting better deposits faster when deposit distance was smaller (see online supplementary material, Figs. S2 and S3), as deposit visits, as well as recruitment, occurred more frequently (for example in Heap2B, the swarms switched to a better deposit after about $25 \mathrm{~min}$ when $D=7 \mathrm{~m}$ and after about $35 \mathrm{~min}$ when $D=13 \mathrm{~m}$ ). However, note that checker swarms were unable to concentrate on the better deposit before the first quality change occurred, as a majority of robots had to abandon the deposit from which they initially foraged before recruitment to a better foraging site could take place. Such a significant abandonment of a deposit could only occur if the deposit was nearly depleted, which did not occur unless the whole swarm foraged from it, or when deposit qualities were changed suddenly by the simulation engine.

Nevertheless, the long-term behaviour of the checkers was much more desirable than that of the beggers and could significantly outperform the control swarms, especially when four deposits were placed in the environment, when the deposit quality change interval was long, or when long-range recruitment signals were used (Figs. 8c, d, 9c, d). However, in some scenarios, the checkers collected less resource than the control swarms. This was the case in all Heap2 scenarios when $T_{Q}=1 \mathrm{~h}$ for both short- and long-range checkers and in the Heap2A scenario when $T_{Q}=2 \mathrm{~h}$ for short-range checkers. The poor performance was caused by the fact that the checkers gradually abandoned a deposit of a worse quality after deposit qualities were changed, which led to a decrease in foraging activity. The robots that stopped 
foraging waited on the dance floor to be recruited or left the base to become scouts, creating a period of time when the swarm could not do any foraging work. Even though the robots eventually did forage from a deposit of a better quality, they often did not have enough time to make up for the loss of foraging time. The period of low foraging activity was especially long for short-range checkers and when the deposit distance was large. For example, it took the swarms up to $35 \mathrm{~min}$ to start foraging from the better deposit in the Heap2B scenario when $D=13 \mathrm{~m}$ and $T_{Q}=1 \mathrm{~h}$ (see online supplementary material, Fig. S2), leading to $38 \%$ decrease in the total amount of resource collected compared to the control swarm (Fig. 8c).

\section{Analysing the value of information}

It is clear that foraging performance in dynamic environments is closely related to how information is obtained and exchanged between robots. While sudden bursts of information transfer can lead to over-commitment to a single deposit, which reduces the chance of acquiring information on alternative deposit sites, a mix of scouting and more controlled information transfer can lead to plasticity. In order to characterise behavioural strategies that are beneficial, it would be useful to have a measure that could quantify the effect of communication.

It is possible to mathematically model swarms using differential equations and then use the model to test simulation parameters and predict the average result (Lerman et al. 2006; Liu and Winfield 2010). It has also been shown that decision-making swarms can be modelled as dynamical systems that exhibit Hopf bifurcations and limit cycles (Pais et al. 2012). Finally, information theoretical measures, such as transfer entropy and local data storage, can be used to show how communication affects the state of an agent based on the states of the other agents it shares information with (Miller et al. 2014).

While mathematical models are often tractable and can generalise well (Martinoli et al. 2004), they are also often complex and difficult to apply in real-life scenarios (McFarland and Spier 1997) where many options exist and where physical interactions substantially influence the resulting swarm behaviour. Inspired by information theoretical approaches, we seek to establish a simple measure, the information value $I$, that works directly from experimental data and represents a quantifiable effect of information transfer between the environment and the swarm and within the swarm itself. This measure should reflect the flow of new information though the system, should be positive when recruitment leads to exploitation of relatively good deposits and should be negative when recruitment causes the system to lower its foraging performance.

The information value $I_{r}$ of a robot is defined as

$$
I_{r}=Q_{\text {new }}-Q_{\text {old }}
$$

where $Q_{\text {new }}$ is the quality of a new deposit the robot finds out about and $Q_{\text {old }}$ is the quality of a deposit it previously foraged from. Note that $Q_{\text {old }}=0$ when a robot has not foraged before. $I_{r}$ is thus equal to $Q_{\text {new }}$ when a scout discovers a new deposit or when a robot that does not posses any information is recruited. In cases when a robot currently foraging from a deposit switches to another, $I_{r}$ can be either positive or negative. For example, if a robot previously foraged from a deposit with $Q_{i}=1.0$ and was recruited to a new deposit with $Q_{i}=0.5, I_{r}=-0.5$. Finally, $I_{r}=0$ for all robots that do not receive any information in a given time step. 
We compare deposit qualities, rather than energy efficiencies, i.e. we do not take the volume left in a deposit or its distance from the base into account. The information value thus only captures utility obtained from a single foraging trip that the robot is guaranteed to make, rather than that of possible future trips. Furthermore, since deposits in our Heap scenarios have the same distance from the base, comparing their qualities is sufficient to identify which one is more profitable.

We obtain the swarm information value $I$ normalised per robot as:

$$
I=\sum_{r=1}^{N_{R}} I_{r} \times \frac{1}{N_{R}}
$$

where $N_{R}$ is the total number of robots. A time series of the swarm (Fig. 10) is obtained by sampling the average value of $I$ over short time intervals (in our case 300 -s intervals). When a swarm working in a dynamic environment is able to repeatedly switch its attention to the current best deposit, the shape of the time series repeats for each quality change interval and has three distinguishable information value regions:

1. A negative region that is caused by recruitment to the previously exploited $\operatorname{deposit}(\mathrm{s})$ that have become worse since the change in deposit quality.

2. A flat region where new and old information coexists in the system. We refer to the negative region and the flat region together as a non-positive region.

3. A positive region that represents recruitment to the newly discovered better-quality deposit(s).

The size (i.e. the area between the curve and the abscissa) of negative regions represents a lag between changes in the environment and changes in the swarm's collective knowledge. This region is usually larger for long-range recruiters and for shorter deposit distances, i.e. when recruitment is stronger (see online supplementary material, Figs. S4-S6). Stronger recruitment tends to result in a larger number of robots to be foraging from the better deposit at the end of a change interval. Immediately after a quality change, there is thus a higher probability of an observer being recruited by a robot that is signalling out-of-date information.

The size of positive regions is influenced by the number of observers that a recruiter holding new information can reach, i.e. by the speed of information transfer through the swarm. It is also larger for long-range recruiters and shorter deposit distances.

The length of the non-positive regions is a combination of the positive impact of information speed and the negative impact of commitment to old information.

When robots deplete and abandon the better deposit(s) before the quality change occurs, as was the case for checkers in Heap2 scenarios when $T_{Q}=2 \mathrm{~h}$, there is no recruitment to a low-quality deposit after the quality change. Therefore, there are no negative regions of $I$ and non-positive regions become very short. On the other hand, when the whole swarm commits to a single deposit and is unable to abandon it throughout the experiment, as was usually the case with long-range beggers, only a single large positive region exists at the beginning of the simulation, followed by zero information value throughout the rest of the run. Using information value, we can thus distinguish three work modes of foraging swarms (Fig. 10):

1. Switching swarms that alternate between non-positive and positive regions and sometimes also show negative regions. These swarms are able to concentrate on deposit(s) of better quality in each quality change interval.

2. Locked swarms that show a large positive region at the beginning of the simulation, but are unable to alter their chosen foraging site for the rest of the run and thus show zero communication value thereafter. 


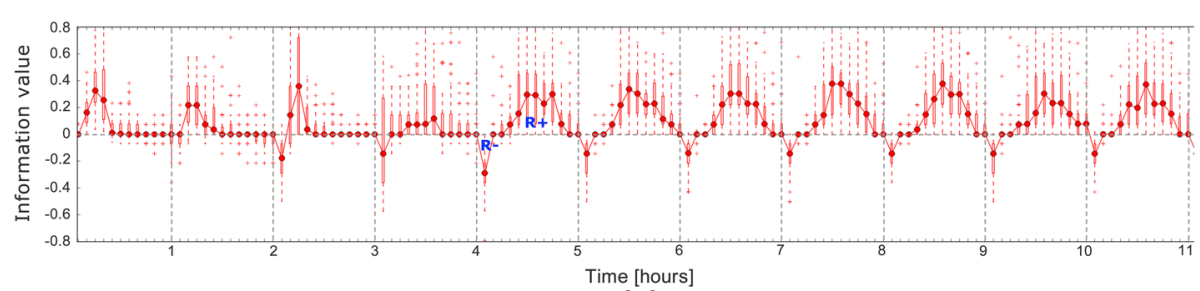

(a)

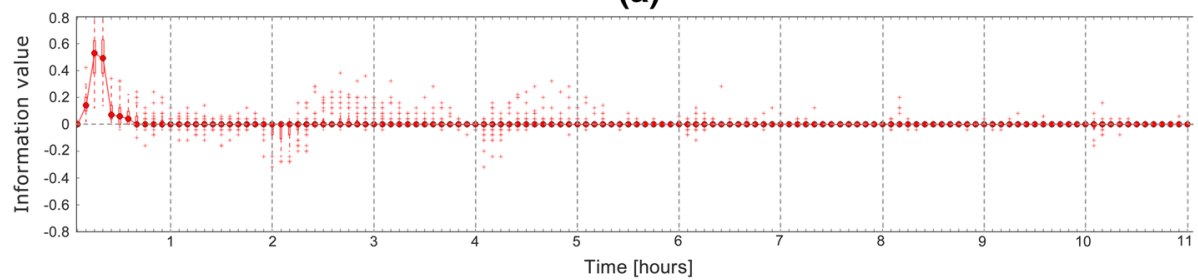

(b)

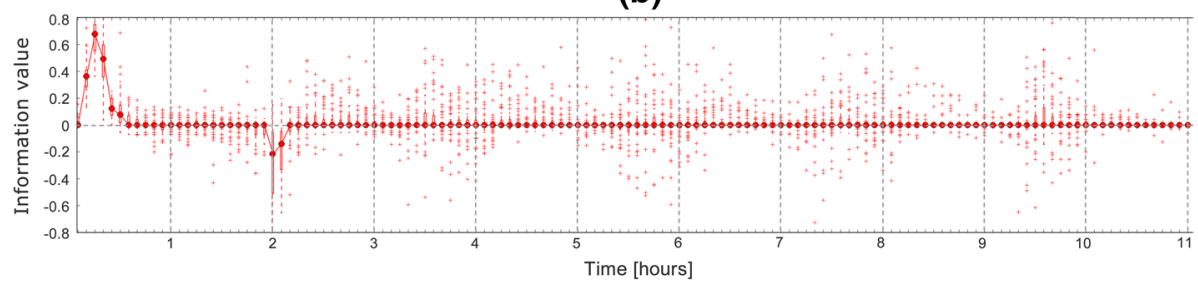

(c)

Fig. 10 An information value time series based on 50 independent runs with 25 robots for a switching swarm: long-range checkers in Heap2B, $D=9 \mathrm{~m}, T_{Q}=1 \mathrm{~h} \mathbf{b}$ locked swarm: long-range beggers in Heap2A, $D=9 \mathrm{~m}, T_{Q}=2 \mathrm{~h} \mathbf{c}$ indecisive swarm: long-range beggers in Heap4B, $D=9 \mathrm{~m}, T_{Q}=2 \mathrm{~h}$. Negative regions $(\mathrm{R}-$ ) and positive regions $(\mathrm{R}+)$ are shown for the switching swarm

3. Indecisive swarms that also have a large positive region at the beginning, but are able to gain new information through scouting or because recruitment does not affect as many robots as in locked swarms. In contrast with switching swarms, rapid information transfer in indecisive swarms prevents the collective from settling on one solution, leading to oscillations around $I=0$. When multiple runs are considered, time series of $I$ has medians of zero and many outliers.

When runs that last several hours and have multiple quality change intervals are considered, locked and indecisive swarms always collect less resource than the control swarms that forage from all deposits with similar proportions. The relative performance of switching swarms depends on a number of factors. As we showed in the previous sections, switching to better deposits can sometimes occur too late or the total amount of loadings can be insufficient, which leads to worse performance. This was, for example, the case for checkers in Heap2 scenarios when $T_{Q}=1 \mathrm{~h}$.

In the following sections, we test our communication strategies further under various experimental perturbations and use the information value measure, $I$, to analyse the results. We show that the switching work mode is more common when information transfer is slower and that measuring $I$ can thus help robot designers to select behavioural parameters that lead to effective plastic self-organisation. 


\begin{tabular}{|c|c|c|c|c|c|}
\hline & & $\begin{array}{c}\text { Short-range } \\
\text { beggers }\end{array}$ & $\begin{array}{c}\text { Long-range } \\
\text { beggers }\end{array}$ & $\begin{array}{c}\text { Short-range } \\
\text { checkers }\end{array}$ & $\begin{array}{c}\text { Long-range } \\
\text { checkers }\end{array}$ \\
\hline \multirow{4}{*}{$\begin{array}{l}\text { Normal } \\
\text { conditions }\end{array}$} & Heap2, TQ = 1h & locked & Iorked & \multirow{4}{*}{ Switching } & \multirow{4}{*}{ Switching } \\
\hline & Heap2, TQ = 2h & LOCKeu & LOCKed & & \\
\hline & Heap4, TQ = 1h & Indecisive & \multirow{2}{*}{ Indecisive } & & \\
\hline & Heap4, TQ = 2h & Switching & & & \\
\hline
\end{tabular}

\begin{tabular}{|c|c|c|c|c|c|}
\hline & & $\begin{array}{c}\text { Short-range } \\
\text { beggers }\end{array}$ & $\begin{array}{c}\text { Long-range } \\
\text { beggers }\end{array}$ & $\begin{array}{c}\text { Short-range } \\
\text { checkers }\end{array}$ & $\begin{array}{c}\text { Long-range } \\
\text { checkers }\end{array}$ \\
\hline \multirow{4}{*}{$\begin{array}{l}\text { Restricted } \\
\text { dance floor } \\
\text { shape }\end{array}$} & Heap2, TQ = 1h & \multirow{3}{*}{$\begin{array}{l}\text { Delayed } \\
\text { switching }\end{array}$} & \multirow{2}{*}{ Locked } & \multirow{4}{*}{ Switching } & \multirow{4}{*}{ Switching } \\
\hline & Heap2, TQ = 2h & & & & \\
\hline & Heap4, TQ = 1h & & \multirow{2}{*}{ Indecisive } & & \\
\hline & Heap4, TQ = 2h & Switching & & & \\
\hline
\end{tabular}

\begin{tabular}{|c|c|c|c|c|c|}
\hline & & $\begin{array}{c}\text { Short-range } \\
\text { beggers }\end{array}$ & $\begin{array}{c}\text { Long-range } \\
\text { beggers }\end{array}$ & $\begin{array}{c}\text { Short-range } \\
\text { checkers }\end{array}$ & $\begin{array}{c}\text { Long-range } \\
\text { checkers }\end{array}$ \\
\hline \multirow{4}{*}{$\begin{array}{l}\text { Increased } \\
\text { pellet } \\
\text { accumulation }\end{array}$} & Heap2, TQ = 1h & \multirow{2}{*}{ Locked } & \multirow{2}{*}{ Locked } & \multirow{2}{*}{ Switching } & \multirow{2}{*}{ Switching } \\
\hline & Heap2, $\mathbf{T Q}=\mathbf{2 h}$ & & & & \\
\hline & Heap4, TQ = $1 \mathrm{~h}$ & Indecisive & \multirow{2}{*}{ Indecisive } & \multirow{2}{*}{ Indecisive } & \multirow{2}{*}{ Indecisive } \\
\hline & Heap4, TQ = 2h & Indecisive & & & \\
\hline
\end{tabular}

\begin{tabular}{|c|c|c|c|c|c|}
\hline & & $\begin{array}{c}\text { Short-range } \\
\text { beggers }\end{array}$ & $\begin{array}{c}\text { Long-range } \\
\text { beggers }\end{array}$ & $\begin{array}{c}\text { Short-range } \\
\text { checkers }\end{array}$ & $\begin{array}{c}\text { Long-range } \\
\text { checkers }\end{array}$ \\
\hline \multirow{4}{*}{$\begin{array}{l}\text { Increased } \\
\text { swarm size }\end{array}$} & Heap2, TQ = 1h & Delayed switching & \multirow{2}{*}{$\begin{array}{l}\text { Delayed } \\
\text { switching }\end{array}$} & \multirow{4}{*}{ Switching } & Delayed switching \\
\hline & Heap2, $\mathrm{TQ}=\mathbf{2 h}$ & Suitching & & & \multirow{3}{*}{ Switching } \\
\hline & Heap4, TQ = $1 \mathrm{~h}$ & switering & \multirow{2}{*}{ Switching } & & \\
\hline & Heap4, TQ = $2 \mathrm{~h}$ & Switching & & & \\
\hline
\end{tabular}

Fig. 11 Work modes exhibited by the swarms under various experimental set-ups. Work modes that differ from those exhibited under normal conditions (see text) are shown in bold

\section{Varying experimental parameters}

It is important to understand how the chosen experimental parameters affect the performance and work modes of our swarms. In this section, we explore the influence of the shape of the dance floor, of the tendency for collected pellets to accumulate and interfere with robot movement, and of the size of the robot swarms. We evaluate how varying these aspects of the foraging scenario affects the behaviour of all four types of swarms (i.e. short-range and longrange beggers, and short-range and long-range checkers) compared to performance under normal conditions (i.e. circular dance floor, no pellet accumulation and 25-robot swarms-see Sects. 5 and 6). We also identify cases in which variation to these aspects of the scenario causes a swarm to transition from one work mode to another (e.g. from switching to indecisive). See Fig. 11 for a summary of all experiments and the work modes exhibited by the swarms.

\subsection{Restricted dance floor shape}

In the previous experiments, a returning forager travelled to the middle of the base before it started recruiting in order to be able to interact with any observer, regardless of direction from which it returned to the base. However, the constraints of a particular foraging scenario 
might require a different approach. For example, we might want to create a recharging area in a part of the recruitment area or otherwise change the shape of the base and thus indirectly alter the way in which robots meet each other and communicate. Information flow throughout a swarm will tend to be influenced by such physical logistics, potentially changing the ability of the swarm to maintain plasticity and thereby influencing performance levels.

In the following experiment, we designated an inaccessible circular area in the middle of the base, making the dance floor doughnut shaped and $35 \mathrm{~cm}$ thick. While the robots could still see other robots across the inaccessible central area, and could thus communicate with them if their communication range allowed for it, they could not move through the central restricted area. Consequently, while undertaking a random walk within the dance floor, a robot tended to remain relatively close to the point at which it first entered the base. The restricted shape of the dance floor also resulted in higher congestion and constrained movement of the robots within and immediately around the dance floor. The congestion was more severe when long-range recruitment was used or when deposits were closer to the base, i.e. when the number of foragers returning to the base at the same time was high. Additionally, the annular shape of the dance floor restricted communication within swarms with short-range recruitment, as it made interactions between robots that foraged from different areas of the environment less probable and prevented information from spreading as easily throughout the swarm.

Although most swarms did not change their work modes, information value analysis showed that restricting dance floor shape decreased the rate at which new information about the best deposit(s) spread through the swarm (see online supplementary material, Figs. S7, S8). For example, the total size of positive regions (i.e. sum of all $I>0$ ) was $40 \%$ smaller for short-range checkers in Heap2s $\left(D=9 \mathrm{~m}\right.$ and $\left.T_{Q}=1 \mathrm{~h}\right)$ compared to normal conditions. The size of positive regions was less affected for long-range checkers (only $28 \%$ smaller), but higher congestion caused the positive regions to appear much later than under normal conditions (usually after 35 instead of $25 \mathrm{~min}$ ).

Short-range beggers were the only swarm that exhibited different work modes compared to the normal conditions (Fig. 11). The decreased probability of interactions between recruiters and observers on the dance floor caused the swarms to escape the locked mode they experienced with a full dance floor in Heap2 scenarios, allowing them to preferentially forage from better deposits. However, clear positive regions of information value were absent while negative regions remained, meaning that the information about new better deposits spread too slowly to be of use. We term this a delayed switching work mode (Fig. 12). The swarms also changed their work mode from indecisive to delayed switching in Heap4 scenarios with $T_{Q}=1 \mathrm{~h}$, as constrained information transfer prevented the confusion that had previously resulted from strong competition between deposits with equivalent energy efficiencies. Despite the fact that, when in the delayed switching mode, short-range beggers were able to alternate between deposits as the deposit quality changed, the foraging performance of the swarms deteriorated compared to normal conditions (Figs. 13a, 14a). First, the total number of foraging trips was smaller due to congestion caused by a smaller dance floor. Second, the delayed switching mode led to a similar number of loadings from deposits of higher and lower quality when the whole run was considered, making the proportion of loadings from the better deposit(s) similar to when the swarms were in the locked or indecisive modes (see online supplementary material, Figs. S9, S10).

The impact of congestion caused by a restrictive dance floor was most clearly observed for long-range beggers. While a recruiter's signal could reach any observer on the dance floor regardless of their relative positions and the work mode of the swarms thus remained locked, 


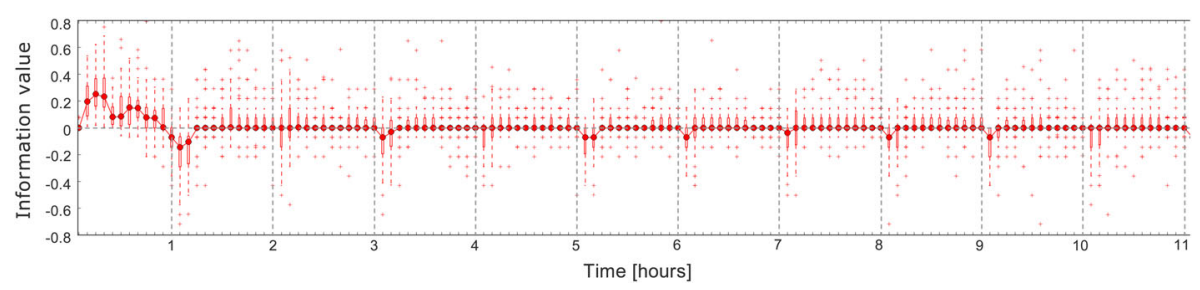

Fig. 12 An information value time series based on 50 independent runs for a delayed switching swarm of 25 short-range beggers in Heap2B, $D=9 \mathrm{~m}, T_{Q}=1 \mathrm{~h}$ with restricted dance floor shape

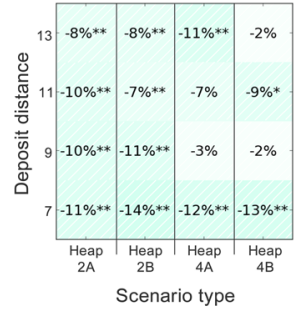

(a)

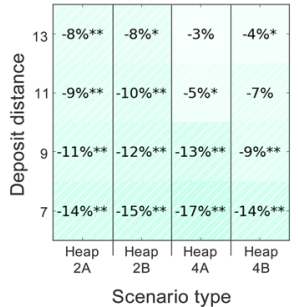

(b)

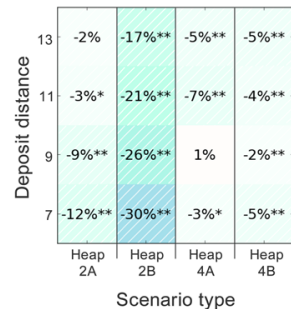

(c)

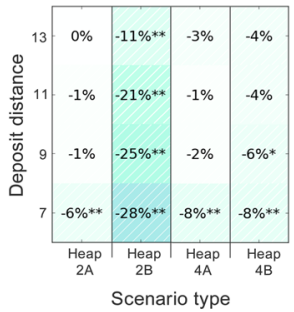

(d)

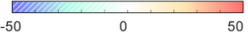

Fig. 13 Difference in the amount of resource collected using an annular dance floor compared to the same communication strategy operating over a full dance floor with a 25 short-range beggers, b 25 long-range beggers, c 25 short-range checkers, d 25 long-range checkers during $1 \mathrm{~h}<T<13 \mathrm{~h}$ of 50 independent runs, using $T_{Q}=1 \mathrm{~h}$. Statistically significant differences are indicated with asterisks (Wilcoxon signed-rank test, $* *=p<0.01, *=p<0.05)$

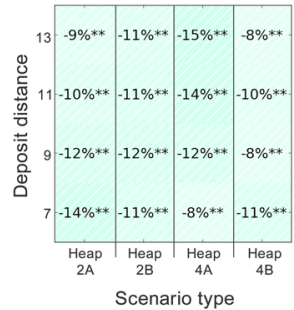

(a)

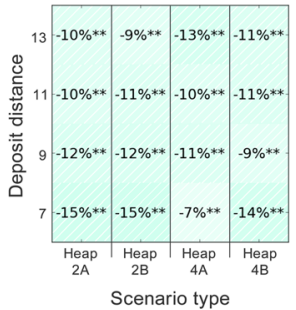

(b)

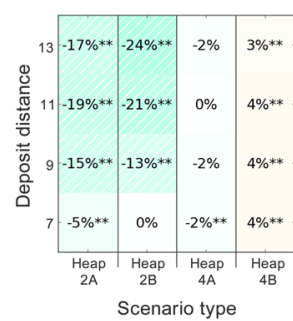

(c)

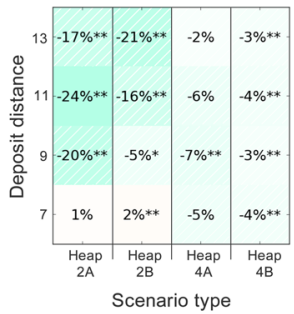

(d)

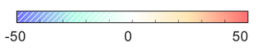

Fig. 14 Difference in the amount of resource collected using an annular dance floor compared to the same communication strategy operating over a full dance floor with a 25 short-range beggers, b 25 long-range beggers, c 25 short-range checkers, d 25 long-range checkers during $2 \mathrm{~h}<T<26 \mathrm{~h}$ of 50 independent runs, using $T_{Q}=2 \mathrm{~h}$. Statistically significant differences are indicated with asterisks (Wilcoxon signed-rank test, $* *=p<0.01, *=p<0.05)$

foraging performance still deteriorated as the robots spent significantly more time avoiding each other (Figs. 13b, 14b).

While the restricted movement of recruiters affected both the short- and long-range checkers negatively in almost all scenarios (Figs. 13c, d, 14c, d), it had a surprisingly positive effect on short-range checkers in Heap4B scenarios when $T_{Q}=2 \mathrm{~h}$ (Fig. 14c). In this case, the impaired ability of robots to recruit each other caused the swarms to forage from the best two 
deposits in parallel and thus to collect a total amount of resource that was higher than under normal conditions.

\subsection{Pellet accumulation}

The material collected by robots in the previous experiments disappeared instantly when it was placed in the unloading bay, i.e. the unloading area handling time $t_{H}=0 \mathrm{~s}$. However, in real-world applications, material would have to be stored somewhere for later use by humans or other robots. It is therefore reasonable to assume that it would accumulate and thus affect the swarm's ability to collect more. To test performance of our swarms under such conditions, we set $t_{H}=\{10,20\}$ s, i.e. pellets dropped by robots could accumulate in the unloading area and create congestion. Since the congestion prevented robots from foraging with maximum efficiency, and from reaching the dance floor in order to communicate, swarms always collected less resources than under normal conditions.

When pellets accumulated, both the negative and positive regions of information value were more flat, while the non-positive regions usually became longer, indicating that limited access to and from the base prevented information transfer. This effect was observed more strongly for long-range recruiters and the higher value of $t_{H}$ (see online supplementary material, Figs. S11, S12), i.e. when more foragers returned to the base at the same time and when pellets remained in the unloading area for a longer period of time. For example, when $t_{H}=20 \mathrm{~s}$, the total size of positive regions (i.e. sum of all $\left.I>0\right)$ in Heap2s $(D=9 \mathrm{~m}$ and $T_{Q}=1 \mathrm{~h}$ ) was $67 \%$ smaller for short-range checkers and $75 \%$ smaller for long-range checkers compared to the normal conditions. The length of non-positive regions decreased by $25 \%$ for short-range checkers and increased by $60 \%$ for long-range checkers.

Since the rate at which the robots communicated was affected, the ability of swarms to switch between deposits was impaired. This caused short-range beggers, short-range checkers and long-range checkers to become indecisive in Heap4 scenarios (Fig. 11). There were no significant changes in work modes observed for long-range beggers, as they already exhibited the locked (in Heap2) and indecisive (in Heap4) work modes under normal conditions.

Pellet accumulation had also an interesting effect on the advantage of long-range over short-range recruitment. For example, under normal conditions, long-range checkers always outperformed short-range checkers in terms of the total amount of resource collected due to their ability to recruit to the best deposit faster. However, when pellets accumulated, this faster recruitment resulted in stronger congestion and in subsequent worse performance compared to short-range checkers in some scenarios (Fig. 15). This was especially true in the Heap2 scenarios when the unloading area handling time $t_{H}=20 \mathrm{~s}$ and when the swarms were given a long time to forage from the selected deposit $\left(T_{Q}=2 \mathrm{~h}\right)$ (Fig. 15d).

\subsection{Increased swarm size}

All previous experiments were performed with swarms of 25 robots. Here, we simulate swarms of 45 and 65 robots.

While short- and long-range beggers previously exhibited the locked work mode in Heap2 scenarios, higher scouting success of the swarm, caused by a higher number of robots that were scouting at the beginning of a simulation run, led to a more even distribution of robots between the deposits. This allowed larger swarms to preferentially forage from better deposits and exhibit the delayed switching work mode, or the switching work mode in the case of short-range beggers when $T_{Q}=2 \mathrm{~h}$ or when $N_{R}=65$ (see Fig. 11 and online supplementary material, Fig. S13). However, the significant delays in recruitment to a better deposit usually 


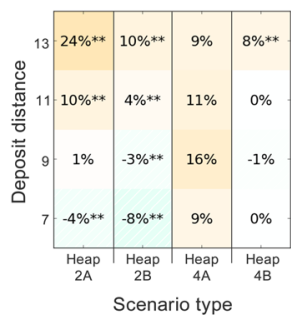

(a)

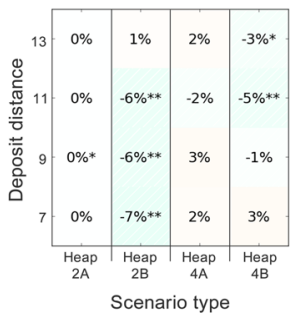

(b)

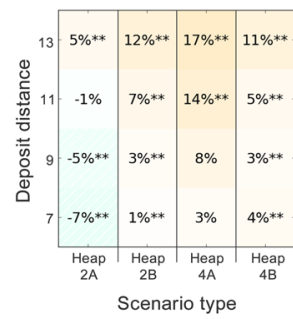

(c)

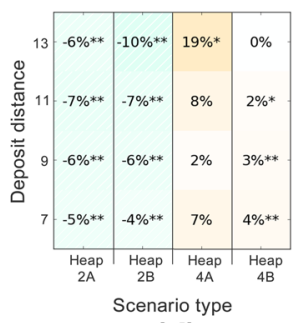

(d)

Fig. 15 Improvement in resource collected using 25 long-range checkers compared to 25 short-range checkers during 50 independent runs with a unloading area handling time $t_{H}=10 \mathrm{~s}, T_{Q}=1 \mathrm{~h}$ and $1 \mathrm{~h}<T<13 \mathrm{~h}$, b $t_{H}=20 \mathrm{~s}, T_{Q}=1 \mathrm{~h}$ and $1 \mathrm{~h}<T<13 \mathrm{~h}, \mathbf{c} t_{H}=10 \mathrm{~s}, T_{Q}=2 \mathrm{~h}$ and $2 \mathrm{~h}<T<26 \mathrm{~h}, \mathbf{d} t_{H}=20 \mathrm{~s}$, $T_{Q}=2 \mathrm{~h}$ and $2 \mathrm{~h}<T<26 \mathrm{~h}$. Statistically significant improvements are indicated with asterisks (Wilcoxon signed-rank test, $* *=p<0.01, *=p<0.05$ )

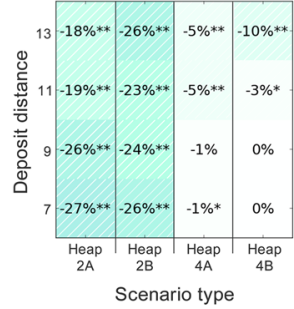

(a)

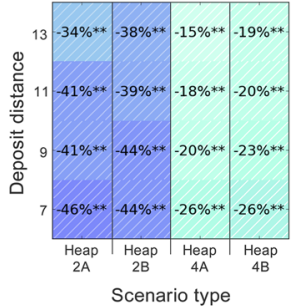

(b)

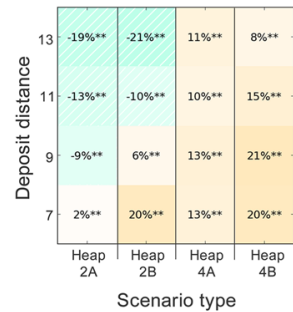

(c)

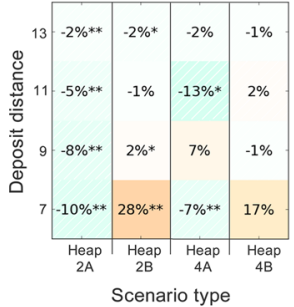

(d)

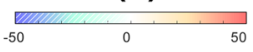

Fig. 16 Improvement in resource collected compared to control (non-switching) swarms of 65 robots using a 65 short-range beggers, b 65 long-range beggers, $\mathbf{c} 65$ short-range checkers, d 65 long-range checkers during $1 \mathrm{~h}<T<13 \mathrm{~h}$ of 50 independent runs, $T_{Q}=1 \mathrm{~h}$. Statistically significant improvements are indicated with asterisks (Wilcoxon signed-rank test, $* *=p<0.01, *=p<0.05$ )

caused a stronger disadvantage for the beggers over the control swarms compared to when the number of robots $N_{R}=25$ (compare Fig. 16a, b with $8 \mathrm{a}$, b and $17 \mathrm{~b}$ with 9b). In Heap4 scenarios, where the beggers were previously indecisive, the swarms were able to achieve the switching mode regardless of the value of $T_{Q}$ (Fig. 11). The switching was effective enough to allow both short- and long-range beggers to outperform the control swarms when $N_{R}=45$. For example, short-range beggers collected $17 \%$ more resources than the control swarms of the same size in Heap4A when $D=7 \mathrm{~m}$ and $N_{R}=45$. However, their advantage largely disappeared when $N_{R}=65$ (Figs. 16a, 17a) due to rapid exploitation of the better foraging sites and consequent ineffective foraging from other deposits, as well as due to increased congestion in the base. The disadvantage of 65-robot swarms was more pronounced for long-range beggers (Figs. 16b, 17b) that shared information with each other faster and thus exploited deposits more rapidly.

Checkers generally retained their ability to switch between deposits when the swarm size increased. The information value of the swarms, normalised per robot, did not change significantly, although the positive regions at the beginning of simulation runs were more prominent compared to when 25 robots were used (see online supplementary material, Fig. S14). This suggests that information spread faster at the beginning of a run, causing the swarms to be able to exhibit periodic switching behaviour sooner. As with beggers, checkers performed 


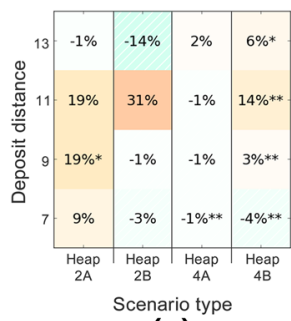

(a)

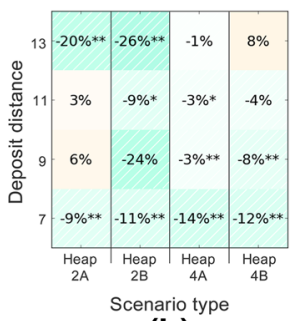

(b)

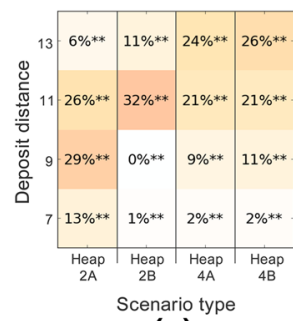

(c)

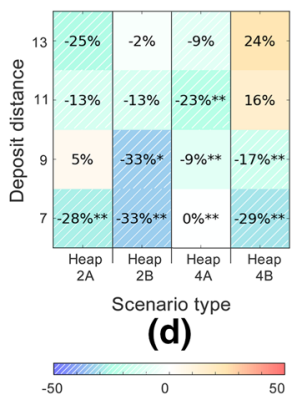

Fig. 17 Improvement in resource collected compared to control (non-switching) swarms of 65 robots using a 65 short-range beggers, b 65 long-range beggers, c 65 short-range checkers, d 65 long-range checkers during $2 \mathrm{~h}<T<26 \mathrm{~h}$ of 50 independent runs, $T_{Q}=2 \mathrm{~h}$. Statistically significant improvements are indicated with asterisks (Wilcoxon signed-rank test, $* *=p<0.01, *=p<0.05$ )

better than the control swarms when $N_{R}=45$. Long-range checkers lost their advantage when $N_{R}=65$ for similar reasons to the beggers (Figs. 16d, 17d). It was also observed that 65-robot swarms of long-range checkers exhibited the delayed switching work mode instead of the switching work mode in Heap2 scenarios when $T_{Q}=1 \mathrm{~h}$ as a result of very significant commitment to a single deposit in each quality change interval and consequent insufficient scouting. Furthermore, there was a large variance in the total amount of collected resource across multiple runs when long-range checkers were used and when $N_{R}=65, T_{Q}=2 \mathrm{~h}$. On the other hand, large swarms of short-range checkers, where information could not spread as quickly, performed well in most of the tested scenarios (Figs. 16c, 17c). This result is not that surprising. If there is an optimal number of robots to find and exploit resources in a given environment, then swarms with long-range communication, where information spreads faster, reach that optimal number sooner and thus also become disadvantaged sooner as $N_{R}$ increases.

\section{Discussion}

\subsection{Summary of results}

We have demonstrated that the nature of communication between individual robots affects a swarm's plasticity and thereby its ability to forage effectively in various types of environment. In particular, the following principles apply:

- Recruitment is most useful when deposits are large and hard to find. Conversely, recruitment can harm foraging performance when resource is distributed over numerous small deposits (see Fig. 4).

- Longer communication range increases foraging performance when deposits are large and hard to find (see Fig. 4), unless rapid exploitation of deposits can cause congestion, e.g. when a lot of the collected material accumulates in the base (see Fig. 15d) or when swarm size is large (see Figs. 16b, d, 17b, d).

- If robots need to choose foraging sites based on deposit energy efficiency in static foraging environments, maximising information flow in the swarm is beneficial as it maximises exploitation of the best sources (see Fig. 5). 
- When environments are dynamic and deposit qualities change over time, both continuous exploitation and exploration of the environment are important. Regulation of information flow, for example by short communication range of robots or by an implementation of individual-based decisions on when to ignore social information, is beneficial as it prevents over-exploitation of a single foraging site (see Figs. 8c, d, 9c, d). A slower information flow thus helps to avoid situations in which a swarm locks into foraging from a single deposit or is unable to discriminate deposits of varying qualities and forages from multiple sources simultaneously.

- Inhibition of social information spread can also be achieved when robots are to some extent physically prevented from meeting and recruiting each other, for example when the shape of the base prevents robots from communicating with those that approach from the opposite side (see online supplementary material, Figs. S7, S8), or when collected material accumulates in the base (see online supplementary material, Figs. S11, S12).

- On the other hand, information is collected and spreads faster when swarm size increases (see online supplementary material, Figs. S13, S14). This can be beneficial especially when robot communication is short range (see Figs. 16c, 17a, c) or in scenarios where smaller swarms cannot alternate between deposits that change their qualities (see Fig. 11).

\subsection{Related work}

Our results are consistent with existing studies in the literature. For example, it has been shown that communication between robots is most beneficial when deposits are scarce (e.g. Liu et al. 2007; Pitonakova et al. 2014) and that short-range communication is most suitable for large robot groups, as too much information can lower task specialisation (Sarker and Dahl 2011). Previous work on the effects of communication range also demonstrated that global information exchange leads to over-commitment to a single resource (Tereshko and Loengarov 2005). Similarly, our swarms of beggers, where information spreads quickly, often found themselves committed to a single source in scenarios with two deposits.

The importance of recruitment when flower patches have a high return has been observed in honey bee colonies (Donaldson-Matasci and Dornhaus 2012). Bees, like our robots in Heap scenarios, can benefit from communicating about a location that affords many successful foraging trips. The fact that recruitment increases the probability of individuals foraging is also important in our Heap scenarios, where robots found it difficult to discover deposits. Similarly, in an agent-based model of a bee colony, recruitment was important in environments with few flower patches (Dornhaus et al. 2006).

A similar scenario has been explored by the HoFoReSim model (Schmickl et al. 2012), where simulated bees foraged from nectar sources, the quality of which changed once during a simulation run. The colony consisted of foragers that brought nectar into the nest and receivers that processed it. If a forager could not find a receiver to unload the nectar to, i.e. when the nest's nectar intake rate was too high for the receivers to handle, the forager did not waggle dance, but instead performed a tremble dance in order to activate additional dormant receivers. The authors argued that the ratio of active receivers to foragers played an important role in the ability of the colony to switch between different sources of nectar when quality changes occurred. Foragers were able to switch to a better source of nectar more quickly when there were relatively few receivers. In this situation, successful foraging overloaded receivers, causing foragers to spend time activating more receivers rather than recruiting more foragers to their foraging sites. This reduction in forager recruitment allowed bees to be less committed to high-quality foraging sites, making responses to a change in 
source quality faster. The role played by scarce receivers was therefore one of information regulation. In our simulation, regulation of information spread is achieved differently, e.g. by shorter communication range or constraints imposed on movement of the robots, but it has the same effect, allowing the swarms to respond to changes in the environment more appropriately. Under this reading, information flow considerations help explain a swarm's ability to exhibit plasticity both in our simulation and in HoFoReSim.

\subsection{Swarm-level plasticity}

In order to analyse foraging swarms, we quantified the value of information transfer and identified the following four work modes that a swarm could reach: switching, delayed switching, locked and indecisive. Swarms in the switching mode could respond to changes in deposit qualities well and usually performed better than swarms in other work modes (for example, see Figs. 8c, d, 9c, d, 9a for Heap4). Delayed switching, locked and indecisive modes were associated with worse levels of performance, although the extent of their disadvantage varied based on the environment and the swarm size.

Figure 18 shows the work modes exhibited by swarms using the different behavioural strategies explored here and the conditions under which they changed from one mode to another. The strategies are ordered from top to bottom by their ability to spread information, i.e. by the size of the positive information value region following the first deposit quality change. We tested the swarms under normal conditions, explored in Sect.6, and under three kinds of perturbation (restricted dance floor shape, pellet accumulation and increased swarm size), explored in Sect.8, giving us four foraging conditions in total. In addition, we explored four different scenario types: Heap2 versus Heap4, and $T_{Q}=1 \mathrm{~h}$ versus $T_{Q}=2 \mathrm{~h}$. We could thus compare the behavioural strategies across a total of $4 \times 4=16$ different experimental set-ups (Fig. 11).

Short-range checkers were in the switching mode in all four scenario types under normal foraging conditions and exhibited the indecisive mode only when material accumulation increased in the Heap4 scenarios with $T_{Q}=1 \mathrm{~h}$ and $T_{Q}=2 \mathrm{~h}$. We can say that the swarms were in the switching mode in 14 out of $16(87.5 \%)$ of cases. Long-range checkers also found themselves in the switching mode in all scenarios under normal conditions, but there were three conditions under which they became either indecisive or delayed. These swarms were in the switching mode in 13 out of $16(81.25 \%)$ cases. Moving along the information speed axis, short-range beggers were in the switching mode in only 5 out of $16(31.25 \%)$ cases, and long-range beggers in only 2 out of $16(12.5 \%)$ cases.

It is clear that rapid information spread often leads to the delayed, locked or indecisive modes, i.e. it prevents plastic self-organisation. However, it is important to point out that foraging performance in static environments was higher when information spread was fast. Furthermore, while the use of long-range recruitment in dynamic environments decreased the number of experimental set-ups where swarms were in the switching mode, it also led to a greater amount of resource being collected when the switching mode did occur and when congestion was not too high due to large swarm size or material accumulation. We could thus say that while using a robot behavioural strategy where information spreads quickly might be desirable in some application scenarios, designers of swarms have to be more certain about foraging conditions like swarm size, material accumulation, number of deposits or the length of the deposit quality change interval. On the other hand, while they were never the best in terms of foraging performance, swarms of short-range checkers, where information spread slowly, exhibited plasticity more commonly, as they retained their ability to switch 


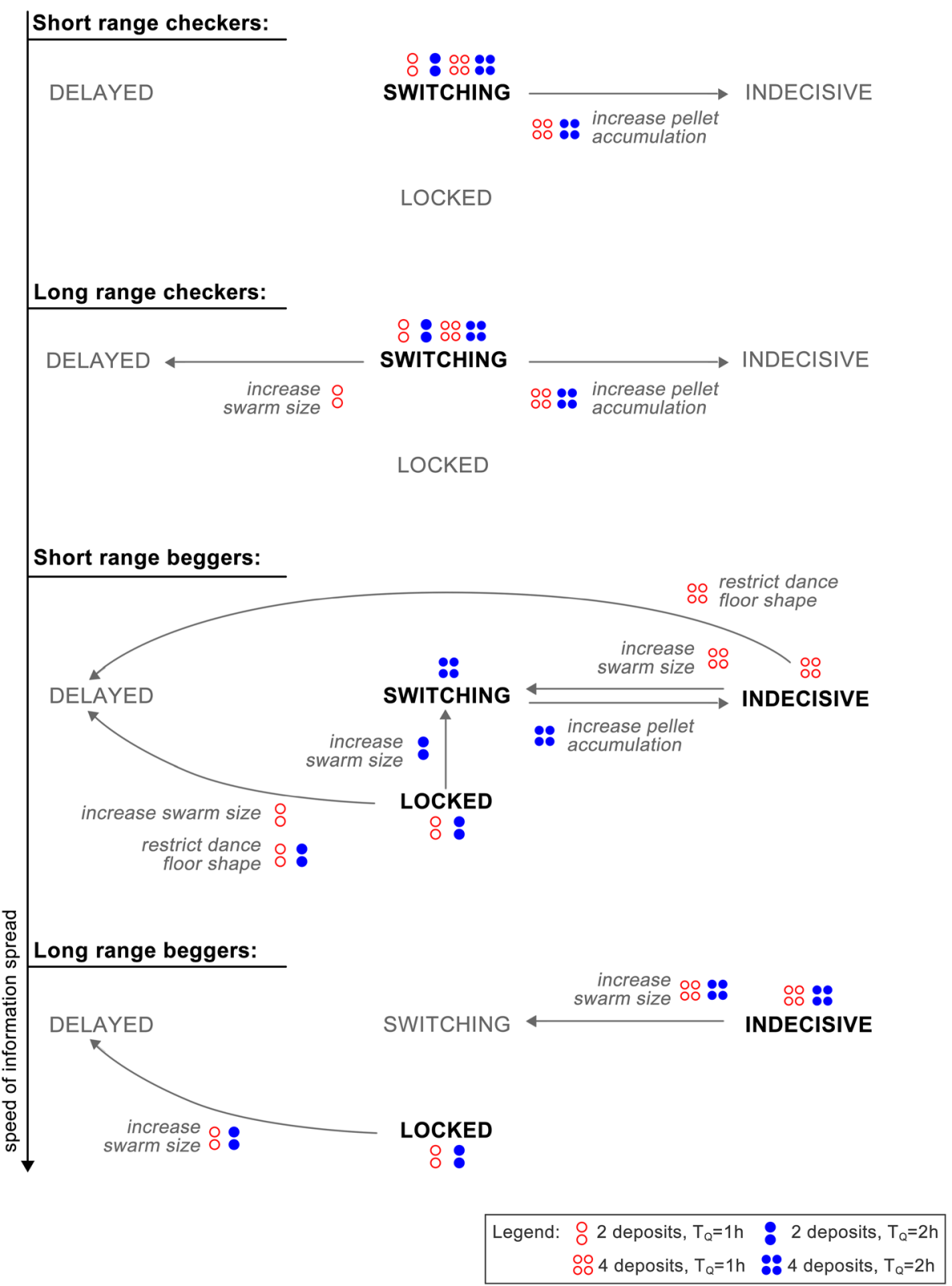

Fig. 18 Work modes and mode transitions exhibited by swarms with different behavioural strategies. The default modes that swarms operated in under normal conditions, i.e. full dance floor, no pellet accumulation, 25 robots (see Sects. 5 and 6), are shown in bold. Scenario markers related to a combination of the number of deposits and the length of deposit quality change interval, $T_{Q}$, are described in the figure legend. The markers are placed above default work modes, and the transition arrows to indicate the scenarios in which they occurred 
between deposits under most tested conditions. Such a strategy would thus be more suitable in unknown or uncontrollable environments.

These findings on the effects of information flow could be applied to a wider range of problems. In particular, the following swarm robotic tasks are the most relevant:

- A number of researchers are interested in collection from different deposit types at the same time (e.g. Balch 1999; Campo and Dorigo 2007). Results here show that inhibition of social information spread, which can be achieved by reducing communication range or restricting the opportunities for communication to take place (in our study, this is effected by restricting the shape of the recruitment area or altering the character of pellet accumulation), can lead to the desired outcome of foraging from different deposits in parallel.

- Communication about resource availability was used in robots that could adapt their resting and waking thresholds based on food density (Liu and Winfield 2010). The speed of information spread, dependent on design decisions about communication type and range, could potentially play an important role in such a task and affect a swarm's flexibility.

- Task allocation has received substantial interest (e.g. Zhang et al. 2007; Sarker and Dahl 2011; Jevtic et al. 2012; Zahadat et al. 2013). It is somewhat similar to deposit selection, as robots need to collectively explore the space of possible work sites and perform work on them. Principles of information transfer could be applied here, for example, by substituting deposit energy efficiency for work site importance or utility during action selection and during transmission of social information. Similarly, information value, introduced in Sect. 7, could be adapted to relate to work site importance rather than deposit quality. We aim to explore this domain in our future work.

- As an alternative to design by hand, swarm behaviour design can be achieved by reinforcement learning (e.g. Pérez-Uribe 2001) or artificial evolution (e.g. Doncieux et al. 2015; Ferrante et al. 2015; Francesca et al. 2015). Knowing what types of communication strategies are beneficial under various conditions or at least which ones are more flexible could minimise the parameter search space for these optimisation algorithms and potentially decrease complexity of the resulting behaviours or save some simulation time.

Finally, it would be possible to utilise information value in an adaptive robot control algorithm in order to make a swarm more autonomous. For example, if robots could identify pathological effects of fast or slow information transfer, they could alter their individual behaviour or their communication range in order to achieve a more effective work mode.

\subsection{Conclusion}

Swarm foraging has many robotic applications and can also be used as a paradigm for other swarm behaviours such as task allocation, labour division or robot dispersion. Social insects like ants and bees give an example of how powerful swarm intelligence can be and how plastic adaptive behaviour can emerge from interactions between relatively limited system parts. However, before we can use robotic swarms in the real world, we need to understand how to design individual agents for reliable collaborative work in dynamic environments.

We argued that the way in which the character of robot-robot communication influences the flow of information through a swarm and the swarm's ability to obtain new information from the environment is an important factor that affects swarm plasticity and, as a consequence, foraging performance. Behavioural strategies that limit the spread of information promote plastic self-organisation and are thus more suitable for unknown or highly dynamic 
environments. We have also demonstrated a method for measuring the value of information that can help us identify such plastic behavioural strategies, not only in foraging swarms but also in a wider range of swarm behaviours where social information is utilised.

Acknowledgments This work was supported by an EPSRC Doctoral Training Centre grant (EP/G03690X/1). All source code and data supporting this study are openly available from the University of Southampton repository at http://dx.doi.org/10.5258/SOTON/385724.

Open Access This article is distributed under the terms of the Creative Commons Attribution 4.0 International License (http://creativecommons.org/licenses/by/4.0/), which permits unrestricted use, distribution, and reproduction in any medium, provided you give appropriate credit to the original author(s) and the source, provide a link to the Creative Commons license, and indicate if changes were made.

\section{References}

Alers, S., Bloembergen, D., Hennes, D., Jong, S. D., Kaisers, M., Lemmens, N.,Tuyls, K., Weiss, G. (2011). Bee-inspired foraging in an embodied swarm. In K. Tumer, P. Yolum, L. Sonenberg, P. Stone (Eds.), Proceedings of the 10th international conference on autonomous agents and multiagent systems (AAMAS 2011), (pp.1311-1312). New York: ACM.

Arab, A., Carollo Blanco, Y., \& Costa-Leonardo, A. M. (2012). Dynamics of foraging and recruitment behavior in the asian subterranean termite coptotermes gestroi (Rhinotermitidae). Psyche: A Journal of Entomology, 2012, Article ID 806782. doi:10.1155/2012/806782.

Arkin, R. C. (1992). Cooperation without communication: Multiagent schema-based robot navigation. Journal of Robotic Systems, 9(3), 351-364.

Balch, T. (1999). The impact of diversity on performance in multi-robot foraging. In O. Etzioni, J. P. Müller, J. M. Bradshaw (Eds.), Proceedings of the third annual conference on autonomous agents, (pp. 92-99). New York: ACM.

Balch, T., \& Arkin, R. C. (1994). Communication in reactive multiagent robotic systems. Autonomous Robots, 1(1), 27-52.

Biesmeijer, J. C., \& De Vries, H. (2001). Exploration and exploitation of food sources by social insect colonies: A revision of the scout-recruit concept. Behavioral Ecology and Sociobiology, 49(2), 89-99.

Bonani, M., Longchamp, V., Magnenat, S., Philippe, R., Burnier, D., Roulet, G., et al. (2010). The marXbot, a miniature mobile robot opening new perspectives for the collective-robotic research. In Proceedings of the 2010 IEEE/RSJ international conference on intelligent robots and systems (IROS 2010), (pp. 4187-4193). Piscataway, NJ: IEEE Press.

Borenstein, J. (1998). Experimental results from internal odometry error correction with the OmniMate mobile robot. IEEE Transactions on Robotics and Automation, 14(6), 963-969.

Campo, A., Dorigo, M. (2007). Efficient multi-foraging in swarm robotics. In F. Almeida e Costa (Ed.), Proceedings of the 9th European conference on advances in artificial life (ECAL 2007), (pp. 696-705) Berlin: Springer.

Campo, A., Gutiérrez, Á., Nouyan, S., Pinciroli, C., Longchamp, V., Garnier, S., et al. (2010). Artificial pheromone for path selection by a foraging swarm of robots. Biological Cybernetics, 103(5), 339-352.

Dai, H. (2009). Adaptive control in swarm robotic systems. The Hilltop Review, 3(1), 54-67.

De Marco, R., \& Farina, W. M. (2001). Changes in food source profitability affect the trophallactic and dance behavior of forager honeybees (Apis mellifera L.). Behavioral Ecology and Sociobiology, 50(5), 441-449.

De Marco, R., \& Farina, W. M. (2003). Trophallaxis in forager honeybees Apis mellifera: Resource uncertainty enhances begging contacts? Journal of Comparative Physiology A, 189, 125-134.

Donaldson-Matasci, M. C., \& Dornhaus, A. (2012). How habitat affects the benefits of communication in collectively foraging honey bees. Behavioral Ecology and Sociobiology, 66(4), 583-592.

Doncieux, S., Bredeche, N., Mouret, J. B., \& Eiben, A. E. (2015). Evolutionary robotics: What, why, and where to. Frontiers in Robotics and AI, 2(4), doi:10.3389/frobt.2015.00004.

Dornhaus, A., Klugl, F., Oechslein, C., Puppe, F., \& Chittka, L. (2006). Benefits of recruitment in honey bees: Effects of ecology and colony size in an individual-based model. Behavioral Ecology, 17(3), 336-344.

Drogoul, A., \& Ferber, J. (1993). From Tom Thumb to the Dockers: Some experiments with foraging robots. In J. Meyer, H. L. Roitblat, \& S. W. Wilson (Eds.), From animals to animats II (pp. 451-459). Cambridge: MIT Press. 
Ducatelle, F., Di Caro, G. A., Pinciroli, C., \& Gambardella, L. M. (2011). Self-organized cooperation between robotic swarms. Swarm Intelligence, 5(2), 73-96.

Farina, W. M., Grüter, C., \& Díaz, P. C. (2005). Social learning of floral odours inside the honeybee hive. Philosophical Transactions of the Royal Society of London, Series B, 272, 1923-1928.

Ferrante, E., Duéñez Guzmán, E. (2013). GESwarm: Grammatical evolution for the automatic synthesis of collective behaviors in swarm robotics. In C. Blum C (Ed.), Proceedings of the fifteenth international conference on genetic and evolutionary computation conference companion (GECCO 2013), (pp. 17-24). ACM.

Ferrante, E., Turgut, A. E., Duéñez-Guzmán, E., Dorigo, M., \& Wenseleers, T. (2015). Evolution of selforganized task specialization in robot swarms. PLoS Computational Biology, 11(8), e1004273. doi:10. 1371/journal.pcbi.1004273.

Francesca, G., Brambilla, M., Brutschy, A., Garattoni, L., Miletitch, R., Podevijn, G., et al. (2015). AutoMoDechocolate: Automatic design of control software for robot swarms. Swarm Intelligence, 9(2-3), 125-152.

Fujisawa, R., Dobata, S., Sugawara, K., \& Matsuno, F. (2014). Designing pheromone communication in swarm robotics: Group foraging behavior mediated by chemical substance. Swarm Intelligence, 8(3), 227-246.

Garnier, S., Tâche, F., Combe, M., Grimal, A., Theraulaz, G. (2007). Alicein pheromone land: An experimental setup for the study of ant-likerobots. In: Proceedings of the 2007 IEEE swarm intelligence symposium (SIS 2007), (pp. 37-44). Piscataway, NJ: IEEE.

Granovskiy, B., Latty, T., Duncan, M., Sumpter, D. J. T., \& Beekman, M. (2012). How dancing honey bees keep track of changes: The role of inspector bees. Behavioral Ecology, 23(3), 588-596.

Gutiérrez, A., Campo, A., Monasterio-Huelin, F., Magdalena, L., \& Dorigo, M. (2010). Collective decisionmaking based on social odometry. Neural Computing and Applications, 19(6), 807-823.

Hoff, N., Wood, R., \& Nagpal, R. (2013). Distributed colony-level algorithm switching for robot swarm foraging. In A. Martinoli, F. Mondada, N. Correll, G. Mermoud, M. A. Hsieh, L. E. Parker, \& K. Stoy (Eds.), Distributed autonomous robotic systems, Springer tracts in advanced robotics (Vol. 83, pp. 417430). Berlin: Springer.

Jevtic, A., Gutiérrez, A., Andina, D., \& Jamshidi, M. (2012). Distributed bees algorithm for task allocation in swarm of robots. IEEE Systems Journal, 6(2), 296-304.

Jones, C., \& Matarić, M. J. (2003). Adaptive division of labor in large-scale minimalist multi-robot systems. In Proceedings of the 2003 IEEE/RSJ international conference on intelligent robots and systems (IROS 2003) (Vol. 2, pp. 1969-1974). Piscataway, NJ: IEEE Press.

Kazama, T., Sugawara, K., Watanabe, T. (2005). Traffic-like movement on a trail of interacting robots with virtual pheromone. In K. Murase, K. Sekiyama, T. Naniwa, N. Kubota, J. Sitte (Eds.), Proceedings of the 3 rd international symposium on autonomous minirobots for research and edutainment (AMiRE 2005), (pp. 383-388). Berlin: Springer.

Krieger, M. J. B., \& Billeter, J. B. (2000). The call of duty: Self-organised task allocation in a population of up to twelve mobile robots. Robotics and Autonomous Systems, 30(1-2), 65-84.

Labella, T. H., Dorigo, \& M., Deneubourg, J.-L. (2004). Efficiency and taskallocation in prey retrieval. In A. J. Ijspeert, M. Murata, \& N. Wakamiya (Eds.), Biologically inspired approaches to advanced information technology, lecture notes in computer science (Vol. 3141, pp. 274-289). Berlin: Springer

Lee, J. H., Ahn, C. W. (2011). Improving energy efficiency in cooperative foraging swarm robots using behavioral model. In R. Abdullah, A. T. Khader, I. Venkat, L. P. Wong, K. G. Subramanian (Eds.), Proceedings of the sixth international conference on bio-inspired computing: theories and applications (BIC-TA'11), (pp. 39-44). Piscataway, NJ: IEEE Press.

Lein, A., Vaughan, R. T. (2009). Adapting to non-uniform resource distributions in robotic swarm foraging through work-site relocation. In: Proceedings of the 2009 IEEE/RSJ international conference on intelligent robots and systems (IROS2009), (pp. 601-606). Piscataway, NJ: IEEE Press.

Lemmens, N., de Jong, S., Tuyls, K., Nowe, A. (2008). Bee behaviour in multi-agent systems. In K. Tuyls, A. Nowe, Z. Guessoum, D. Kudenko (Eds.), Adaptive agents and multi-agent systems III. Adaptation and multi-agent learning, lecture notes in computer science, (Vol. 4865, pp. 145-156). Berlin: Springer.

Lerman, K., Jones, C., Galstyan, A., \& Mataric, M. J. (2006). Analysis of dynamic task allocation in multi-robot systems. The International Journal of Robotics Research, 25, 225-242.

Liu, W., \& Winfield, A. F. (2010). Modelling and optimisation of adaptive foraging in swarm robotic systems. The International Journal of Robotics Research, 29(14), 1743-1760.

Liu, W., Winfield, A. F., Sa, J., Chen, J., \& Dou, L. (2007). Strategies for energy optimisation in a swarm of foraging robots. In E. Sahin, W. M. Spears, A. F. Winfield (Eds.), Swarm robotics, lecture notes in computer science, (Vol. 4433, pp. 14-26). Berlin: Springer.

Martinoli, A., Easton, K., \& Agassounon, W. (2004). Modeling swarm robotic systems: A case study in collaborative distributed manipulation. The International Journal of Robotics Research, 23(4), 415-436. 
Mayet, R., Roberz, J., Schmickl, T., \& Crailsheim, K. (2010). Antbots: A feasible visual emulation of pheromone trails for swarm robots. In M. Dorigo, M. Birattari, G. A. Di Caro, R. Doursat, A. P. Engelbrecht, D. Floreano, L. M. Gambardella, R. Groß, E. Sahin, H. Sayama, T. Stützle (Eds.), Swarm intelligence, lecture notes in computer science, (Vol. 6234, pp. 84-94). Berlin: Springer.

McFarland, D., \& Spier, E. (1997). Basic cycles, utility and opportunism in self-sufficient robots. Robotics and Autonomous Systems, 20, 179-190.

Miletitch, R., Trianni, V., Campo, A., \& Dorigo, M. (2013). Information aggregation mechanisms in social odometry. In P. Liò, O. Miglino, G. Nicosia, S. Nolfi, M. Pavone (Eds.), Proceedings of the twelfth European conference on the synthesis and simulation of living systems (ECAL 2013), (pp. 102-109). Cambridge, MA: MIT Press.

Miller, J. M., Wang, X. R., Lizier, J. T., Prokopenko, M., \& Rossi, L. F. (2014). Measuring information dynamics in swarms. In M. Prokopenko (Ed.), Guided self-organisation: inception, emergence, complexity and computation (Vol. 9, pp. 343-364). Berlin: Springer.

Nouyan, S., Groß, R., Bonani, M., Mondada, F., \& Dorigo, M. (2009). Teamwork in self-organized robot colonies. IEEE Transactions on Evolutionary Computation, 13(4), 695-711.

Pais, D., Caicedo-Núnez, C. H., \& Leonard, N. E. (2012). Hopf bifurcations and limit cycles in evolutionary network dynamics. SIAM Journal on Applied Dynamical Systems, 11(4), 1754-1784.

Parunak, H., \& Brueckner, S. A. (2004). Engineering swarmings systems. In F. Bergenti, M. P. Gleizes, \& F. Zambonelli (Eds.), Methodologies and software engineering for agent systems, multiagent systems, artificial societies, and simulated organizations (Vol. 11, pp. 341-376). Berlin: Springer.

Pérez-Uribe, A. (2001). Using a time-delay actor-critic neural architecture with dopamine-like reinforcement signal for learning in autonomous robots. In S. Wermter, J. Austin, D. Willshaw (Eds.), Emergent neural computational architectures based on neuroscience, lecture notes in computer science, (Vol. 2036, pp. 522-533). Berlin: Springer.

Pinciroli, C., Trianni, V., O’Grady, R., Pini, G., Brutschy, A., Brambilla, M., et al. (2012). ARGoS: A modular, parallel, multi-engine simulator for multi-robot systems. Swarm Intelligence, 6(4), 271-295.

Pini, G., Brutschy, A., Pinciroli, C., Dorigo, M., \& Birattari, M. (2013). Autonomous task partitioning in robot foraging: An approach based on cost estimation. Adaptive Behavior, 21(2), 118-136.

Pitonakova, L., Crowder, R., \& Bullock, S. (2014). Understanding the role of recruitment in collective robot foraging. In H. Lipson, H. Sayama, J. Rieffel, S. Risi, \& R. Doursat (Eds.), Proceedings of the fourteenth international conference on the synthesis and simulation of living systems (ALIFE 14) (pp. 264-271). Cambridge, MA: MIT Press.

Ranjbar-Sahraei, B., Weiss, G., \& Nakisaei, A. (2012). A multi-robot coverage approach based on stigmergic communication. In I. J. Timm \& C. Guttmann (Eds.), Multiagent system technologies, lecture notes in computer science, (Vol. 7598, pp. 126-138). Berlin: Springer.

Reynolds, A. M., \& Rhodes, C. J. (2009). The Lévy flight paradigm: Random search patterns and mechanisms. Ecology, 90(4), 877-887.

Ribeiro, P. L., Helene, A. F., Xavier, G., Navas, C., \& Ribeiro, F. L. (2009). Ants can learn to forage on one-way trails. PloS one, 4(4), e5024.

Russell, R.A. (1999). Ant trails-An example for robots to follow? In: Proceedings of the 1999 IEEE international conference on robotics and automation, (Vol. 4, pp. 2698 - 2703). Piscataway, NJ: IEEE Press.

Sarker, M. O. F., \& Dahl, T. S. (2011). Bio-Inspired communication for self-regulated multi-robot systems. In T. Yasuda (Ed.), Multi-robot systems, trends and development (pp. 367-392). InTech. doi:10.5772/ 13104.

Schmickl, T., \& Crailsheim, K. (2008). Throphallaxis within a robotic swarm: Bio-inspired communication among robots in a swarm. Autonomous Robots, 25(1), 171-188.

Schmickl, T., \& Hamann, H. (2010). BEECLUST: A swarm algorithm derived from honeybees. In Y. Xiao (Ed.), Bio-Inspired Computing and Networking (pp. 95-137). Abingdon: Routledge.

Schmickl, T., Möslinger, C., \& Crailsheim, K. (2007). Collective perception in a robot swarm. In E. Sahin, W. M. Spears, \& A. F. Winfield (Eds.), Swarm robotics, lecture notes in computer science, (Vol. 4433, pp. 144-157). Berlin: Springer.

Schmickl, T., Thenius, R., \& Crailsheim, K. (2012). Swarm-intelligent foraging in honeybees: Benefits and costs of task-partitioning and environmental fluctuations. Neural Computing and Applications, 21(2), 251-268.

Seeley, T. D. (1994). Honey bee foragers as sensory units of their colonies. Behavioral Ecology and Sociobiology, 34(1), 51-62.

Seeley, T. D., \& Morse, Ra. (1976). The nest of the honey bee (Apis mellifera L.). Insectes Sociaux, 23, 495-512.

Seeley, T. D., Camazine, S., \& Sneyd, J. (1991). Collective decision-making in honey bees: How colonles choose among nectar sources. Behavioral Ecology and Sociobiology, 28, 277-290. 
Seeley, T. D., Visscher, P. K., Schlegel, T., Hogan, P. M., Franks, N. R., \& Marshall, Ja R. (2012). Stop signals provide cross inhibition in collective decision-making by honeybee swarms. Science, 335(6064), $108-111$.

Serugendo, G. M., Gleizes, M. P., \& Karageorgos, A. (2006). Self-organisation and emergence in MAS: An overview self-organisation. Informatica, 30, 45-54.

Shell, D. A., \& Mataric, M. J. (2006). On foraging strategies for large-scale multi-robot systems. Proceedings of the 2006 IEEE/RSJ international conference on intelligent robots and systems (IROS 2006) (pp. 27172723). Piscataway, NJ: IEEE Press.

Sumpter, D. J. T., \& Beekman, M. (2003). From nonlinearity to optimality: Pheromone trail foraging by ants. Animal Behaviour, 66(2), 273-280.

Tereshko, V., \& Loengarov, A. (2005). Collective decision-making in honey bee foraging dynamics. Computing and Information Systems Journal, 9(3), 1-7.

Thenius, R., Schmickl, T., \& Crailsheim, K. (2008). Optimisation of a honeybee-colony's energetics via social learning based on queuing delays. Connection Science, 20(2-3), 193-210.

Ulam, P., \& Balch, T. (2004). Using optimal foraging models to evaluate learned robotic foraging behavior. Adaptive Behavior, 12(4), 213-222.

Valdastri, P., Corradi, P., Menciassi, A., Schmickl, T., Crailsheim, K., Seyfried, J., et al. (2006). Micromanipulation, communication and swarm intelligence issues in a swarm microrobotic platform. Robotics and Autonomous Systems, 54(10), 789-804.

Valentini, G., Hamann, H., \& Dorigo, M. (2014). Self-organized collective decision making: The weighted voter model. InProceedings of the 13th international conference on autonomous agents and multiagent systems (AAMAS 2014) (pp. 45-52). New York: ACM.

von Frisch, K. (1967). The dance language and orientation of bees. Cambridge: Harvard University Press.

Winfield, A. F. (2009). Towards an engineering science of robot foraging. In H. Asama, H. Kurokawa, \& K. Sekiyama (Eds.), Distributed Autonomous Robotic Systems 8 (pp. 185-192). Berlin: Springer.

Zahadat, P., Crailsheim, K., \& Schmickl, T. (2013). Social inhibition manages division of labour in artificial swarm systems. In P. Liò, O. Miglino, G. Nicosia, S. Nolfi, \& M. Pavone (Eds.), Proceedings of the twelfth European conference on the synthesis and simulation of living systems (ECAL 2013) (pp. 609616). Cambridge, MA: MIT Press.

Zhang, D., Xie, G., Yu, J., \& Wang, L. (2007). Adaptive task assignment for multiple mobile robots via swarm intelligence approach. Robotics and Autonomous Systems, 55(7), 572-588. 